\title{
On the Index of \\ Constant Mean Curvature 1 Surfaces in Hyperbolic Space
}

\section{Levi Lopes De Lima, Wayne Rossman}

\author{
To Manfredo do Carmo \\ on the occasion of his seventieth birthday
}

\begin{abstract}
We show that the index of a constant mean curvature 1 surface in hyperbolic 3-space is completely determined by the compact Riemann surface and secondary Gauss map that represent it in Bryant's Weierstrass representation. We give three applications of this observation. Firstly, it allows us to explicitly compute the index of the catenoid cousins and some other examples. Secondly, it allows us to be able to apply a method similar to that of Choe (using Killing vector fields on minimal surfaces in Euclidean 3-space) to our case as well, resulting in lower bounds of index for other examples. And thirdly, it allows us to give a more direct proof of the result by do Carmo and Silveira that if a constant mean curvature 1 surface in hyperbolic 3-space has finite total curvature, then it has finite index. Finally, we show that for any constant mean curvature 1 surfaces in hyperbolic 3 -space that have been constructed via a correspondence to minimal surfaces in Euclidean 3-space, we can take advantage of this correspondence to find a lower bound for its index.
\end{abstract}

1. Introduction. In a seminal paper $[\mathrm{By}], \mathrm{R}$. Bryant has shown that the geometry of surfaces with constant mean curvature 1 in hyperbolic 3 -space $\mathbb{H}^{3}(-1)$ has many similarities with the geometry of minimal surfaces in Euclidean space $\mathbb{R}^{3}$. It was shown in particular that such surfaces admit a Weierstrass representation in terms of certain holomorphic data (see Section 3 below for details). A detailed analysis of this representation has allowed the construction of many complete examples ([UY1], [RUY]).

It is well known that constant mean curvature surfaces in $\mathbb{R}^{3}$ and $\mathbb{H}^{3}$ can be characterized as critical points for the area functional, under compactly sup- 
ported variations. (Recall that in the constant mean curvature nonzero case, only volume preserving variations are allowed.) Regarding these variational problems, in the cases of complete minimal surfaces in $\mathbb{R}^{3}$ and complete constant mean curvature 1 surfaces in $\mathbb{H}^{3}$, it is known that the only stable objects are planes and horospheres $[\mathrm{CP}],[\mathrm{Si}]$. This makes all the more interesting the study of surfaces of finite index, namely, surfaces for which the dimension of the space of area decreasing variations is finite. A fundamental result regarding this point is due to Fischer-Colbrie [FC], who has shown that a minimal surface in $\mathbb{R}^{3}$ has finite index if and only if its total curvature is finite. (In regard to this, see also [G].) In fact, Fischer-Colbrie's analysis allows us to obtain explicit estimates for the index of concrete examples with finite total curvature. Recall that any such surface is conformally equivalent to a compact Riemann surface $\Sigma$ punctured at finitely many points corresponding to the ends of the original surface. Moreover, the Gauss map of $M$ extends meromorphically across the punctures defining a meromorphic map $g: \Sigma \rightarrow S^{2}$. Then, it follows from Fischer-Colbrie's arguments that the index of $M$ coincides with the index of the Schrödinger operator on $\Sigma$ defined by

$$
\mathcal{L}=\triangle-|d g|^{2} .
$$

Here, $\triangle$ and $|d g|$ are computed relatively to any metric on $\Sigma$ that is conformally equivalent to the original metric on $M$.

The purpose of this paper is to extend this circle of ideas to constant mean curvature 1 surfaces in $\mathbb{H}^{3}(-1)$. In this case, the role played by the map $g$ is replaced by the so-called secondary Gauss map $G$ (we describe $G$ below; it is a certain multivalued map that comes from the Bryant-Weierstrass representation). It so happens that if $M \in \mathbb{H}^{3}(-1)$ has constant mean curvature 1 and finite total curvature, then $M$ is also conformally finite, but it is no longer true, in general, that $G$ extends meromorphically across the ends. This means, as we shall see, that the analysis necessary for studying the index in the $\mathbb{H}^{3}(-1)$ case is much more involved than in the $\mathbb{R}^{3}$ case. More precisely, let $d s^{2}$ and $K$ denote the induced metric and the Gaussian curvature in both cases. A common feature here is that $K \leq 0$ and vanishes only at isolated points (unless $M$ is either a plane or a horosphere) and that $d \bar{s}^{2}=-K d s^{2}$ is a spherical pseudo-metric on $M$ with conical singularities at these points.

A crucial point here is to determine the behavior of $d \bar{s}^{2}$ at an end of $M$. To this effect, let $z=(x, y)$ be a conformal parameter around some end so that the end corresponds to $z=0$. Note that, since $M$ is complete, $d s^{2}$ certainly becomes infinite when $z \rightarrow 0$. Also, since the surface has finite total curvature, the limiting value of $-K$ as $z \rightarrow 0$ is zero. So at first sight, it is unclear what the behavior of $d \bar{s}^{2}=-K d s^{2}$ is at the ends. It is well known, however, that in the minimal case we can choose $z$ so that

$$
d \bar{s}^{2}=\frac{4\left|g^{\prime}\right|^{2}}{\left(1+|g|^{2}\right)^{2}}|d z|^{2},
$$

where primes denote derivative with respect to $z$. Moreover, since $g$ extends meromorphically across the ends, we can assume $g \approx z^{\ell}, \ell \in \mathbb{Z}^{+}$, so that $d \bar{s}^{2}$ is 
bounded around the end. One can now take advantage of this fact when one does the analysis necessary for examining the index of the minimal case, eventually obtaining Fischer-Colbrie's results. In the hyperbolic case, we shall compute below that

$$
d \bar{s}^{2}=\frac{4\left|G^{\prime}\right|^{2}}{\left(1+|G|^{2}\right)^{2}}|d z|^{2} .
$$

But now we can only assume that $G(z) \approx z^{\mu}$, for some $\mu>0$ depending only upon the end. In particular, if $0<\mu<1$ for some end, $d \bar{s}^{2}$ is not bounded at this end and the analysis for the minimal case does not apply to this situation.

Doing the necessary extra analysis is the heart of this paper. More precisely, we show that the canonical form for $d \bar{s}^{2}$ (just above) around the ends implies that the Sobolev space $\bar{H}^{1}=H_{d \bar{s}^{2}}^{1}$ is compactly embedded in $L_{d \bar{s}^{2}}^{2}$ (Lemma 4.4). Once this has been established, it is an easy matter to use standard variational methods to define $\operatorname{Ind}(\Sigma)$ as being the index of a certain operator $\bar{L}$ defined on $\Sigma$ and corresponding to the Schrödinger operator $\mathcal{L}$ in the minimal case (Section 5 ). It follows easily from the construction that $\operatorname{Ind}_{u}(M) \leq \operatorname{Ind}(\Sigma)$, where $\operatorname{Ind}_{u}(M)$ denotes the unconstrained index of $M$, namely, the index as computed for not necessarily volume preserving variations (it follows from our arguments that $\operatorname{Ind}(M)$ and $\operatorname{Ind}_{u}(M)$ differ at most by 1 , so that computing $\operatorname{Ind}_{u}(M)$ takes us a great deal of the way toward computing $\operatorname{Ind}(M)$, our ultimate concern here). Furthermore, using standard results in elliptic regularity theory, we show that the eigenfunctions of $\bar{L}$ extend continuously across the ends (Lemma 5.2). This extra regularity property enables us to show that $\operatorname{Ind}(\Sigma) \leq \operatorname{Ind}_{u}(M)$, after an argument due to Fischer-Colbrie (Lemma 5.3).

Once this analysis is done, we find that we have an alternate proof of the result by do Carmo and Silveira [CS] that if a constant mean curvature 1 surface in $\mathbb{H}^{3}(-1)$ has finite total curvature, then it has finite index (Corollary 5.2). The advantage of our way of proving this result is that it gives us tools that allow us to compute explicit bounds on index for some concrete examples. For some surfaces we can even compute the index exactly.

For example, using methods similar to those of Nayatani [N1], we can compute the index of the catenoid cousins and the Enneper cousins of higher winding order, as well as some other examples described by Umehara and Yamada [UY1] (Section 6). Our results about the index of these examples yield some surprising differences from the index of minimal surfaces in $\mathbb{R}^{3}$. For example, unlike the minimal catenoid in $\mathbb{R}^{3}$, the catenoid cousins in $\mathbb{H}^{3}(-1)$ can have arbitrarily high index (Theorem 6.1). Also, although the only minimal surfaces in $\mathbb{R}^{3}$ with index 1 are the catenoid and Enneper's surface, there are many more examples of index 1 surfaces in the hyperbolic case (final remark of Section 6).

As another example, using methods similar to those of Choe [Cho], we can compute lower bounds for many constant mean curvature 1 surfaces in $\mathbb{H}^{3}(-1)$ (Theorem 7.1). We find lower bounds for genus $1 n$-noid cousins (Corollary 7.3), and for genus $k$ Costa surface cousins (Corollary 7.1). And, in general, for those constant mean curvature 1 surfaces that are constructed via a deformation 
method $[R U Y]$ from minimal surfaces in $\mathbb{R}^{3}$, we can find a lower bound for index (Theorem 8.1).

The second author owes special thanks to Shin Nayatani for many helpful discussions. Thanks are also due to Pierre Berard, Etienne Sandier, Shin Kato, and David Goldstein.

2. Definition of index. Let $\Phi: M \rightarrow M^{3}(a)$ be an isometric immersion of a 2-dimensional manifold $M$ into a complete simply-connected 3-dimensional manifold $M^{3}(a)$ with constant sectional curvature $a$. Let $\vec{N}$ be a unit normal vector field on $\Phi(M)$ (we write $\Phi^{*} \vec{N}$ simply as $\vec{N}$ defined on $M$ ). Let $\Phi(t)$ be a smooth variation of immersions for $t \in(-\varepsilon, \varepsilon)$ so that $\Phi(0)=\Phi$. Assume that the variation has compact support. We can assume that the corresponding variation vector field at time $t=0$ is $u \vec{N}, u \in C_{0}^{\infty}(M)$. Let $A(t)$ be the area of $\Phi(t)(M)$ and $H$ be the mean curvature of $\Phi(M)$. The first variational formula $([\mathrm{L}])$ is

$$
\left.\frac{d A}{d t}\right|_{t=0}=-\int_{M}\langle n H \vec{N}, u \vec{N}\rangle d A,
$$

where $\langle$,$\rangle and d A$ are the metric and area form on $M$ induced by the immersion $\Phi$. If $H$ is constant, then $A^{\prime}(0)=-n H \int_{M} u d A$. Let $V(t)$ be the volume of $\Phi(t)(M)$, then $V^{\prime}(0)=\int_{M} u d A$. A variation is said to be volume preserving if $\int_{M} u d A=0$. It follows that $\Phi(M)$ is critical for area amongst all volume preserving variations.

The second variation formula for volume preserving variations ([Che], [Si], $[\mathrm{L}])$ is

$$
\left.\frac{d^{2} A}{d t^{2}}\right|_{t=0}:=\int_{M}\left\{|\nabla u|^{2}-2\left(2 a+2 H^{2}-K\right) u^{2}\right\} d A,
$$

where $K$ is the Gaussian curvature on $M$. Since we will be investigating surfaces of constant mean curvature 1 in hyperbolic space with constant sectional curvature -1 , we will restrict ourselves to the case $a=-1$ and $H=1$, so

$$
\left.\frac{d^{2} A}{d t^{2}}\right|_{t=0}=\int_{M}\left\{|\nabla u|^{2}+2 K u^{2}\right\} d A .
$$

This formula is the same for both minimal surfaces in $\mathbb{R}^{3}:=M^{3}(0)$ and constant mean curvature 1 surfaces in $\mathbb{H}^{3}:=M^{3}(-1)$, giving us our first indication of the close relationship between these two types of surfaces. Another indication of this close relationship is the Weierstrass representations described in the next section.

The index $\operatorname{Ind}(M)$ is the maximum possible dimension of a subspace of volume preserving variation functions in $C_{0}^{\infty}(M)$ on which

$$
\left.\frac{d^{2} A}{d t^{2}}\right|_{t=0}<0
$$


The purpose of this paper is to estimate $\operatorname{Ind}(M)$.

We define $\operatorname{Ind}_{u}(M)$ as the maximum possible dimension of a subspace of (not necessarily volume preserving) variation functions in $C_{0}^{\infty}(M)$ on which the above $\left(d^{2} A\right) /\left.\left(d t^{2}\right)\right|_{t=0}<0$. (The subscript $u$ stands for "unconstrained index".) Clearly, $\operatorname{Ind}_{u}(M) \geq \operatorname{Ind}(M)$. We will show later that also $\operatorname{Ind}_{u}(M)-1 \leq$ $\operatorname{Ind}(M)$. The methods we use in this paper allow us to compute $\operatorname{Ind}_{u}(M)$, but what we really want to compute is $\operatorname{Ind}(M)$. However, these two indices can differ by at most 1 , so computing $\operatorname{Ind}_{u}(M)$ means that we know $\operatorname{Ind}(M)$ must be either $\operatorname{Ind}_{u}(M)$ or $\operatorname{Ind}_{u}(M)-1$.

3. The Weierstrass representation. Both minimal surfaces in $\mathbb{R}^{3}$ and constant mean curvature 1 surfaces in $\mathbb{H}^{3}$ can be described parametrically by a pair of meromorphic functions on a Riemann surface, via a Weierstrass representation. First we describe the well-known Weierstrass representation for minimal surfaces in $\mathbb{R}^{3}$. We will incorporate into this representation the fact that any complete minimal surface of finite total curvature is conformally equivalent to a Riemann surface $\Sigma$ with a finite number of points $\left\{p_{j}\right\}_{j=1}^{k} \subset \Sigma$ removed ([O]):

Lemma 3.1 Let $\Sigma$ be a Riemann surface. Let $\left\{p_{j}\right\}_{j=1}^{k} \subset \Sigma$ be a finite number of points, which will represent the ends of the minimal surface defined in this lemma. Let $z_{0}$ be a fixed point in $\Sigma \backslash\left\{p_{j}\right\}$. Let $g$ be a meromorphic function from $\Sigma \backslash\left\{p_{j}\right\}$ to $\mathbb{C}$. Let $f$ be a holomorphic function from $\Sigma \backslash\left\{p_{j}\right\}$ to $\mathbb{C}$. Assume that, for any point in $\Sigma \backslash\left\{p_{j}\right\}, f$ has a zero of order $2 k$ at some point if and only if $g$ has a pole of order $k$ at that point, and assume that $f$ has no other zeroes on $\Sigma \backslash\left\{p_{j}\right\}$. Then

$$
\Phi(z)=\operatorname{Re} \int_{z_{0}}^{z}\left(\begin{array}{c}
\left(1-g^{2}\right) f d \zeta \\
i\left(1+g^{2}\right) f d \zeta \\
2 g f d \zeta
\end{array}\right)
$$

is a conformal minimal immersion of the universal cover $\widetilde{\Sigma \backslash\left\{p_{j}\right\}}$ of $\Sigma \backslash\left\{p_{j}\right\}$ into $\mathbb{R}^{3}$. Furthermore, any complete minimal surface with finite total curvature in $\mathbb{R}^{3}$ can be represented in this way.

The map $g$ can be geometrically interpreted as the stereographic projection of the Gauss map. The first and second fundamental forms and the intrinsic Gaussian curvature for the surface $\Phi$ are

$$
\begin{aligned}
& d s^{2}=(1+g \bar{g})^{2} f d z \cdot \overline{f d z}, \\
& \mathrm{II}=-2 \operatorname{Re}(Q), \\
& K=-4\left(\frac{\left|g^{\prime}\right|}{|f|\left(1+|g|^{2}\right)^{2}}\right)^{2},
\end{aligned}
$$

where the Hopf differential $Q$ is defined to be $Q=g^{\prime} f d z^{2}$. 
To make a surface of finite total curvature (i.e. $\int_{\Sigma}-K d A<+\infty$, which is necessary to make a surface of finite index $[\mathrm{FC}]$ ) we must choose $f$ and $g$ so that $\Phi$ is well defined on $\Sigma \backslash\left\{p_{j}\right\}$ itself. Usually this involves adjusting some real parameters in the descriptions of $f$ and $g$ and $\Sigma \backslash\left\{p_{j}\right\}$ so that the real part of the above integral about any nontrivial loop in $\Sigma \backslash\left\{p_{j}\right\}$ is zero.

We now describe a Weierstrass type representation for constant mean curvature $c$ surfaces in $\mathbb{H}^{3}\left(-c^{2}\right):=M^{3}\left(-c^{2}\right)$. This result is a composite of several results that are found in [By], [UY3], [UY4].

Lemma 3.2 Let $\Sigma, \Sigma \backslash\left\{p_{j}\right\}, z_{0}$, $f$, and $g$ be the same as in the previous lemma. Choose a null holomorphic immersion $F: \widetilde{\Sigma \backslash\left\{p_{j}\right\}} \rightarrow S L(2, \mathbb{C})$ so that $F\left(z_{0}\right)$ is the identity matrix and so that $F$ satisfies

$$
F^{-1} d F=c\left(\begin{array}{ll}
g & -g^{2} \\
1 & -g
\end{array}\right) f d z .
$$

Then $\Phi: \widetilde{\Sigma \backslash\left\{p_{j}\right\}} \rightarrow H^{3}\left(-c^{2}\right)$ defined by

$$
\Phi=\frac{1}{c} F^{-1} \bar{F}^{-1}
$$

is a conformal constant mean curvature c immersion into $\mathbb{H}^{3}\left(-c^{2}\right)$ with the Hermitian model. Furthermore, any constant mean curvature c surface with finite total curvature in $\mathbb{H}^{3}\left(-c^{2}\right)$ can be represented in this way.

We call $g$ the hyperbolic Gauss map of $\Phi$. As its name suggests, the map $g(z)$ has a geometric interpretation for this case as well. It is the image of the composition of two maps. The first map is from each point on the surface to the point at the sphere at infinity in the Poincare model which is at the opposite end of the oriented perpendicular geodesic ray starting at the point $z$ on the surface. The second map is stereographic projection of the sphere at infinity to the complex plane $\mathbb{C}[\mathrm{By}]$. The first and second fundamental forms and the intrinsic Gaussian curvature of the surface are

$$
\begin{aligned}
& d s^{2}=(1+G \bar{G})^{2} \frac{f g^{\prime}}{G^{\prime}} \overline{\left(\frac{f g^{\prime}}{G^{\prime}}\right)} d z \overline{d z}, \\
& \mathrm{II}=-2 \operatorname{Re}(Q)+c d s^{2}, \\
& K=-4\left(\frac{\left|G^{\prime}\right|^{2}}{\left|g^{\prime}\right||f|\left(1+|G|^{2}\right)^{2}}\right)^{2},
\end{aligned}
$$

where in this case the Hopf differential is $Q=-f g^{\prime} d z^{2}$ (the sign change in $Q$ is due to the fact that we are considering the "dual" surface; see [UY4] for an explanation of this), and where $G$ is defined as the multi-valued meromorphic function

$$
G=\frac{d F_{11}}{d F_{21}}=\frac{d F_{12}}{d F_{22}}
$$


on $\Sigma \backslash\left\{p_{j}\right\}$, with $F=\left(F_{i j}\right)_{i, j=1,2}$. The reason that $G$ is multi-valued is that $F$ itself can be multi-valued on $\Sigma \backslash\left\{p_{j}\right\}$ (even if $\Phi$ is well defined on $\Sigma \backslash\left\{p_{j}\right\}$ itself). The function $G$ is called the secondary Gauss map of $\Phi$ ([By]).

In the lemma above, we have changed the notation slightly from the notation used in [By] and [RUY], because we wish to use the same symbol " $g$ " both for the map $g$ used in the Weierstrass representation for minimal surfaces in $\mathbb{R}^{3}$ and for the hyperbolic Gauss map used in the Weierstrass representation for constant mean curvature surfaces in $\mathbb{H}^{3}$. And we further wish to give a separate notation " $G$ " for the secondary Gauss map used in the hyperbolic case. We do this to emphasize that, in relation to their geometric interpretations, the " $g$ " in the Euclidean case is more closely related to the hyperbolic Gauss map " $g$ " in the $\mathbb{H}^{3}$ case than to the secondary Gauss map " $G$ " (as we will see in Section 6).

In order for $\Phi$ to be well defined on $\Sigma \backslash\left\{p_{j}\right\}$ itself, it is sufficient and necessary that $F$ satisfy a condition called the $S U(2)$-condition. Note that if one travels about a nontrivial loop in $\Sigma \backslash\left\{p_{j}\right\}$, then $F \rightarrow B F$, where $B \in S L(2, \mathbb{C})$. If for every loop in $\Sigma \backslash\left\{p_{j}\right\}$, the resulting matrix $B$ satisfies $B \in S U(2)$, then the $S U(2)$-condition is satisfied. If $B \in S U(2)$, then $F^{-1}{\overline{F^{-1}}}^{t}=(B F)^{-1}{\overline{(B F)^{-1}}}^{t}$, so it follows that if the $S U(2)$-condition holds, then $\Phi$ is well defined on $\Sigma \backslash\left\{p_{j}\right\}$ itself. When $F \rightarrow B F$, we have the following effect on the secondary Gauss map:

$$
G \rightarrow \frac{b_{11} G+b_{12}}{b_{21} G+b_{22}}, \text { for } B=\left(\begin{array}{ll}
b_{11} & b_{12} \\
b_{21} & b_{22}
\end{array}\right) \in S U(2) .
$$

We now state some known facts, which when taken together, show that constant mean curvature 1 surfaces in $\mathbb{H}^{3}$ and minimal surfaces in $\mathbb{R}^{3}$ are very closely related. These facts provide the motivation for the results in Sections 7 and 8 of this paper:

- It was shown in [UY2] that if $f$ and $g$ and $\Sigma \backslash\left\{p_{j}\right\}$ are fixed, then as $c \rightarrow 0$, the constant mean curvature $c$ surfaces $\Phi$ in $\mathbb{H}^{3}\left(-c^{2}\right)$ converge to a minimal surface in $\mathbb{R}^{3}$. This can be sensed from the fact that $G \rightarrow g$ and $B \rightarrow$ identity as $c \rightarrow 0$ (which follow directly from equation 3.1), and hence the above first and second fundamental forms for the constant mean curvature $c$ surfaces $\Phi$ converge to the fundamental forms for a minimal surface as $c \rightarrow 0$ (up to a sign change in II - a change of orientation).

- It was shown in [RUY] that a finite total curvature minimal surface in $\mathbb{R}^{3}$ satisfying certain nondegeneracy and symmetry conditions (these conditions are fairly general and include most known examples) can be deformed into a constant mean curvature $c$ surface in $\mathbb{H}^{3}\left(-c^{2}\right)$ for $c \approx 0$, so that $\Sigma$, $f$, and $g$ are the same, up to a slight adjustment of the real parameters that are used to solve the period problem. The deformed surface might not have finite total curvature, but it will be of the same topological type as the minimal surface, and it will have the same reflectional symmetries as the minimal surface. 
- Consider the Poincaré model for $\mathbb{H}^{3}\left(-c^{2}\right)$ for $c \approx 0$. It is a round ball in $\mathbb{R}^{3}$ centered at the origin with Euclidean radius $1 / c$ endowed with a complete radially-symmetric metric

$$
d s_{c}^{2}=\frac{4 \sum d x_{i}^{2}}{\left(1-c^{2} \sum x_{i}^{2}\right)^{2}}
$$

of constant sectional curvature $-c^{2}$. Contracting this model by a factor of $c$, we obtain a map to the Poincaré model for $\mathbb{H}^{3}$. Under this mapping, constant mean curvature $c$ surfaces are mapped to constant mean curvature 1 surfaces. Thus the problem of existence of constant mean curvature $c$ surfaces in $\mathbb{H}^{3}\left(-c^{2}\right)$ for $c \approx 0$ is equivalent to the problem of existence of constant mean curvature 1 surfaces in $\mathbb{H}^{3}$. Furthermore, under this mapping, the area form on the constant mean curvature $c$ surface is changed only by a constant factor $c^{2}$ : If $d A_{c}$ is the area form on the constant mean curvature $c$ surface, and $d A_{1}$ is the area form on the constant mean curvature 1 surface, then $d A_{c}=c^{2} d A_{1}$. Hence a variation that reduces area on the constant mean curvature $c$ surface is mapped to a variation that reduces area on the constant mean curvature 1 surface (and vice-versa). Hence this mapping preserves the index.

4. Showing that $\bar{H}^{1}$ is compactly contained in $L_{d \bar{s}^{2}}^{2}$. We now consider $M$ to be a complete constant mean curvature 1 surface in $\mathbb{H}^{3}$ with finite total curvature. We will assume the surface is not a horosphere. (Assuming that the surface is not a horosphere will not add any extra conditions to our index results, since the index of the horosphere is known to be zero [Si].) Suppose that $M$ has Weierstrass representation $\Phi: \Sigma \backslash\left\{p_{j}\right\} \rightarrow M$ with Riemann surface $\Sigma$ and functions $f, g: \Sigma \rightarrow \mathbb{C}$, and that $G$ is the secondary Gauss map. Let $d s^{2}$ be the complete metric on $M$ pulled back to $\Sigma$. Note that $M$ is conformally equivalent to $\Sigma$ with a finite number of points $\left\{p_{j}\right\}$ removed; each removed point $p_{j}$ corresponds to an end of $M$. So $d s^{2}$ is defined on $\Sigma \backslash\left\{p_{j}\right\}$. Let $d \bar{s}^{2}=G^{*} d s_{S^{2}}^{2}=-K d s^{2}$ be the singular pull back metric of the canonical metric on $S^{2}$ via the secondary Gauss map $G$, defined on $\Sigma \backslash\left\{p_{j}\right\}$, but with isolated singularities where $K=0$. We let $d \tilde{s}^{2}$ be a conformal nonsingular metric defined on $\Sigma$. Any choice for $d \tilde{s}^{2}$ will suffice, provided it is conformally equivalent to $d s^{2}$ on $\Sigma \backslash\left\{p_{j}\right\}$. Let $d A$ (resp. $\left.d \bar{A}, d \tilde{A}\right)$ and $\nabla(\operatorname{resp} . \bar{\nabla}, \tilde{\nabla})$ and $\triangle($ resp. $\bar{\triangle}, \tilde{\triangle})$ be the area form and gradient and Laplacian on $\Sigma$ with respect to the metric $d s^{2}$ (resp. $\left.d \bar{s}^{2}, d \tilde{s}^{2}\right)$.

We choose the sign of the Laplacian so that $\int_{\Omega}|\nabla u|^{2}=+\int_{\Omega} u \triangle u$ for any $u \in C_{0}^{\infty}(\Omega)$. (Thus, for example, the Laplacian on the standard Euclidean plane $\mathbb{R}^{2}$ will be $-\partial^{2} / \partial x^{2}-\partial^{2} / \partial y^{2}$.) So if $u \in C_{0}^{\infty}(\Omega)$ satisfies $\triangle u=\lambda u$ for some constant $\lambda$, where $\Omega$ is a region in $\Sigma$, then $\int_{\Omega}|\nabla u|^{2}=\int_{\Omega} u \triangle u=\lambda \int_{\Omega} u^{2}$ and so $\lambda \geq 0$. Thus our convention for the sign of the Laplacian implies that the eigenvalues of the Laplacian will be nonnegative. 
We now list some easily determined facts that will be used throughout this and the next section. We can define $|d G|_{d s^{2}}^{2}\left(\right.$ resp. $\left.|d G|_{d \bar{s}^{2}}^{2},|d G|_{d \tilde{s}^{2}}^{2}\right)$ by

$$
\begin{aligned}
|d G|_{d s^{2}}^{2} & =\sum_{j=1}^{2}\left\langle d G\left(e_{j}\right), d G\left(e_{j}\right)\right\rangle_{d s_{S^{2}}^{2}} \\
\text { resp. } \quad|d G|_{d \bar{s}^{2}}^{2} & =\sum_{j=1}^{2}\left\langle d G\left(\bar{e}_{j}\right), d G\left(\bar{e}_{j}\right)\right\rangle_{d s_{S^{2}}^{2}}, \\
|d G|_{d \tilde{s}^{2}}^{2} & \left.=\sum_{j=1}^{2}\left\langle d G\left(\tilde{e}_{j}\right), d G\left(\tilde{e}_{j}\right)\right\rangle_{d s_{S^{2}}^{2}}, \quad\right)
\end{aligned}
$$

where $d G$ is the tangent map of $G$ and $\left\{e_{1}, e_{2}\right\}$ (resp. $\left\{\bar{e}_{1}, \bar{e}_{2}\right\},\left\{\tilde{e}_{1}, \tilde{e}_{2}\right\}$ ) is an orthonormal basis of vector fields with respect to the metric $d s^{2}$ (resp. $d \bar{s}^{2}, d \tilde{s}^{2}$ ). The following hold:

- $d \bar{s}^{2}=-K d s^{2}, d \bar{A}=-K d A, \triangle=-K \bar{\triangle}$

- $d \bar{s}^{2}=\frac{1}{2}|d G|_{d \bar{s}^{2}}^{2} d \bar{s}^{2}=\frac{1}{2}|d G|_{d s^{2}}^{2} d s^{2}=\frac{1}{2}|d G|_{d \tilde{s}^{2}}^{2} d \tilde{s}^{2} \quad$ (conformal invariance).

We now consider the variation described in the second section with variation vector field $u \vec{N}$ on $M$ at time $t=0$. Since $d^{2} A /\left.d t^{2}\right|_{t=0}$ obviously depends on $u$, we will write it as $d^{2} A /\left.d t^{2}\right|_{t=0}(u)$. In the next lemma, we will consider $\Sigma$ and $u$ to be fixed, but we consider whether or not $d^{2} A /\left.d t^{2}\right|_{t=0}(u)$ depends on $G, g$, and $f$.

We now state a crucial computation - it is crucial because is explains why the pull-back of the metric on the sphere via the map $G$ plays such a dominant role in computing $\operatorname{Ind}(M)$, and explains why the operators $L$ and $\bar{L}$ (defined later) are somehow "the same" operator:

$$
\begin{aligned}
\left.\frac{d^{2} A}{d t^{2}}\right|_{t=0}(u) & =\int_{\Sigma}\left\{|\nabla u|^{2}+2 K u^{2}\right\} d A=\int_{\Sigma}\left\{u \triangle u+2 K u^{2}\right\} d A \\
& =\int_{\Sigma}\left\{-u K \bar{\triangle} u+2 K u^{2}\right\} d A \\
& =\int_{\Sigma}\left\{-u \bar{\triangle} u+2 u^{2}\right\} K d A \\
& =\int_{\Sigma}\left\{u \bar{\triangle} u-2 u^{2}\right\} d \bar{A} .
\end{aligned}
$$

Since the integrand $\left\{u \bar{\triangle} u-2 u^{2}\right\} d \bar{A}$ is completely determined by the pull-back of the spherical metric via the map $G$, we know that $d^{2} A /\left.d t^{2}\right|_{t=0}(u)$ depends only on $G$, and does not depend on $g$ and $f$.

Lemma $4.1 d^{2} A /\left.d t^{2}\right|_{t=0}(u)$ is completely independent of $f$ and $g$. It does depend on $G$, but not on the choice of value of the multi-valued $G$. 
Proof. As noted above, $d^{2} A /\left.d t^{2}\right|_{t=0}(u)$ depends only on $G$, not on $g$ and $f$. Clearly, $d^{2} A /\left.d t^{2}\right|_{t=0}(u)$ does depend on $G$, but to show that it does not depend on the choice of value of the multi-valued $G$, we first show that the first fundamental form is independent of the $S U(2)$-condition. Let $\hat{f}=-g^{\prime} f / G^{\prime}$. Travelling about a loop in $\Sigma$ corresponding to a homologically nontrivial loop in $M$, we have $F \rightarrow B F$ for some $B \in \mathrm{SU}(2)$, and as we saw before,

$$
G \rightarrow \frac{b_{11} G+b_{12}}{b_{21} G+b_{22}},
$$

where $b_{i j}$ are the entries of $B$. Thus travelling about the loop makes the transformation $G^{\prime} \rightarrow G^{\prime} /\left(b_{21} G+b_{22}\right)^{2}$, and since $f$ and $g$ are left unchanged (and therefore $g^{\prime}$ is also unchanged), it follows that $\hat{f} \rightarrow \hat{f}\left(b_{21} G+b_{22}\right)^{2}$. Now we consider the conformal factor $\left.\hat{f} \overline{\hat{f}}(1+G \bar{G})^{2}\right)^{2}$ in the first fundamental form. Denoting $\left(b_{11} G+b_{12}\right) /\left(b_{21} G+b_{22}\right)$ as $B \cdot G$, we see that

$$
\begin{aligned}
\left.\hat{f} \overline{\hat{f}}(1+G \bar{G})^{2}\right)^{2} & \rightarrow\left(b_{21} G+b_{22}\right)^{2} \hat{\hat{f}} \overline{\left(b_{21} G+b_{22}\right)^{2} \hat{f}}(1+(B \cdot G)(\overline{B \cdot G}))^{2} \\
& \left.=\hat{f} \overline{\hat{f}}(1+G \bar{G})^{2}\right)^{2}
\end{aligned}
$$

since $b_{11}=\overline{b_{22}}$ and $b_{12}=-\overline{b_{21}}$.

Therefore $\langle$,$\rangle is independent of the \mathrm{SU}(2)$-condition, and therefore $\nabla u$ and $d A$ are independent of the $S U(2)$-condition, since they are determined by the first fundamental form. And since $K$ depends only on the first fundamental form, $K$ is also independent of the $S U(2)$-condition. We conclude that $d^{2} A /\left.d t^{2}\right|_{t=0}(u)$ is independent of the $S U(2)$-condition. Thus $d^{2} A /\left.d t^{2}\right|_{t=0}(u)$ is well defined even though $G$ is multi-valued.

For any $p \geq 2$, let $L_{d \tilde{s}^{2}}^{p}(\Omega)$ (resp. $L_{d \bar{s}^{2}}^{p}(\Omega)$ ) be the space of measurable functions $f$ on $\Omega \subset \Sigma$ such that $\int_{\Omega}|f(x)|_{d \tilde{s}^{2}}^{p} d \tilde{A}<\infty\left(\operatorname{resp} . \int_{\Omega}|f(x)|_{d \bar{s}^{2}}^{p} d \bar{A}<\infty\right)$. In the case that $\Omega=\Sigma$, we may write simply $L_{d \tilde{s}^{2}}^{p}$ (resp. $L_{d \bar{s}^{2}}^{p}$ ) instead of $L_{d \tilde{s}^{2}}^{p}(\Sigma)$ (resp. $\left.L_{d \bar{s}^{2}}^{p}(\Sigma)\right)$.

We now begin to work toward a proof that $\bar{H}^{1}$ is compactly contained in $L_{d \bar{s}^{2}}^{2}$.

Lemma 4.2 If $p$ is sufficiently large, then $L_{d \tilde{s}^{2}}^{p}$ is continuously contained in both $L_{d \tilde{s}^{2}}^{2}$ and $L_{d \bar{s}^{2}}^{2}$.

Proof. Since $\Sigma$ is compact, $L_{d \tilde{s}^{2}}^{p}$ is continuously contained in $L_{d \tilde{s}^{2}}^{2}$ for all $p \geq 2$. (See, for example, [GT], equation (7.8)).

As for the second assertion, consider a point $p_{j} \in \Sigma$ representing an end of the complete surface. Let $U_{j}$ be a small neighborhood of $p_{j}$. We may choose $d \tilde{s}^{2}$ so that $d \tilde{s}^{2}=d x^{2}+d y^{2}=4 d z d \bar{z}$ on $U_{j}$. We now show that locally on $U_{j}$,

$$
d \bar{s}^{2} \approx 4 \mu^{2} \frac{r^{2 \mu-2}}{\left(1+r^{2 \mu}\right)^{2}} d \tilde{s}^{2}
$$


with $r=\sqrt{x^{2}+y^{2}}$. (The symbol " $\approx$ " means that for functions $a(z), b(z)$ defined in a neighborhood of $z=0, a(z) \approx b(z)$ if for all $\varepsilon>0$, there exists a $\delta>0$ such that $|z|<\delta$ implies $|a(z) / b(z)-1|<\varepsilon$.) The relation above follows from the fact that locally near an end we can make the following normalization: we can choose the complex coordinate $z$ on $U_{j}$ so that the end $p_{j}$ is at $z=0$. By the previous lemma, we may change $G$ to $\left(b_{11} G+b_{12}\right) /\left(b_{21} G+b_{22}\right)$ for any $B=\left\{b_{i j}\right\} \in$ $\mathrm{SU}(2)$, without affecting the second variation formula. We may choose $B$ so that $\left(\left(b_{11} G+b_{12}\right) /\left(b_{21} G+b_{22}\right)\right)(z=0)=0$. Hence we may assume that $G(0)=0$. We then have that $G=z^{\mu} \hat{G}$, where $\hat{G}$ is a holomorphic function in a neighborhood of $z=0$ such that $\hat{G}(0) \neq 0$, for some $\mu \in \mathbb{R}^{+}$, where $z=x+i y$ [UY1]. Changing $z$ to $\hat{G}(0)^{-1 / \mu} z$ if necessary, we may assume that $\hat{G}(0)=1$.

The point corresponding to $G(z)$ under the inverse of stereographic projection is

$$
\mathcal{G}=\left(\mathcal{G}_{1}, \mathcal{G}_{2}, \mathcal{G}_{3}\right)=\frac{1}{|G|^{2}+1}\left(2 \operatorname{Re}(G), 2 \operatorname{Im}(G),|G|^{2}-1\right)
$$

Note that for any real-valued function $f: \mathbb{C} \rightarrow \mathbb{R}$, we have $f_{z}=\frac{1}{2}\left(f_{x}-i f_{y}\right)$, so $\left|f_{z}\right|^{2}=\frac{1}{4}\left(\left|f_{x}\right|^{2}+\left|f_{y}\right|^{2}\right)$. For any complex-valued holomorphic function $f: \mathbb{C} \rightarrow \mathbb{C}$, we have

$$
\begin{aligned}
\frac{\partial}{\partial z}(\operatorname{Re}(f)) & =\frac{\partial}{\partial z} \frac{1}{2}(f+\bar{f})=\frac{1}{2} f_{z}, \\
\frac{\partial}{\partial z}(\operatorname{Im}(f)) & =\frac{\partial}{\partial z} \frac{i}{2}(\bar{f}-f)=\frac{-i}{2} f_{z}, \\
\frac{\partial}{\partial z}(f \bar{f}) & =f_{z} \bar{f}+f \bar{f}_{z}=\bar{f} f_{z} .
\end{aligned}
$$

Using these properties and the fact that $G=z^{\mu} \hat{G}$, we have

$$
\begin{aligned}
|d G|_{d \tilde{s}^{2}}^{2}=|d \mathcal{G}|_{d \tilde{s}^{2}}^{2} & =\sum_{i=1}^{3}\left|\nabla \mathcal{G}_{i}\right|^{2}=\sum_{i=1}^{3}\left(\left|\left(\mathcal{G}_{i}\right)_{x}\right|^{2}+\left|\left(\mathcal{G}_{i}\right)_{y}\right|^{2}\right) \\
& =\sum_{i=1}^{3} 4\left|\left(\mathcal{G}_{i}\right)_{z}\right|^{2} \\
& =\frac{8 G_{z} \overline{G_{z}}}{(1+G \bar{G})^{2}} \approx \frac{8 \mu^{2} r^{2 \mu-2}}{\left(1+r^{2 \mu}\right)^{2}} .
\end{aligned}
$$

And thus it follows that

$$
d \bar{s}^{2} \approx 4 \mu^{2} \frac{r^{2 \mu-2}}{\left(1+r^{2 \mu}\right)^{2}} d \tilde{s}^{2}
$$

on $U_{j}$.

Suppose $u \in L_{d \tilde{s}^{2}}^{p}\left(U_{j}\right)$. By the Hölder inequality we have

$$
\int_{U_{j}} u^{2} d \bar{A}=\int_{U_{j}} u^{2} \frac{1}{2}|d G|_{d \tilde{s}^{2}}^{2} d \tilde{A} \leq A^{2 / p} B^{1 / q}
$$


where $A=\int_{U_{j}} u^{p} d \tilde{A}$ and $B<c \int_{U_{j}} r^{(2 \mu-2) q} d \tilde{A}$, with $2 / p+1 / q=1$ and $c>0$ some finite constant. If $q$ is close enough to 1 , then $B$ is finite, since $\mu>0$ and $d \tilde{A}$ has the local expression $d \tilde{A}=r d r d \theta$ in polar coordinates. So there exists a constant $k_{j}$ such that $\|u\|_{L_{d \bar{s}^{2}}^{2}\left(U_{j}\right)} \leq k_{j}\|u\|_{L_{d \tilde{s}^{2}}^{p}\left(U_{j}\right)}$ for each $j$.

On $\Sigma \backslash\left\{\bigcup U_{j}\right\}, d \bar{s}^{2}$ is bounded. So it is clear from the Hölder inequality that there exists a constant $k_{0}$ such that

$$
\|u\|_{L_{d \bar{s}^{2}}^{2}\left(\Sigma \backslash\left\{\bigcup U_{j}\right\}\right)} \leq k_{0}\|u\|_{L_{d \tilde{s}^{2}}^{p}\left(\Sigma \backslash\left\{\bigcup U_{j}\right\}\right)} .
$$

Let $k=\max \left\{k_{0}, k_{j}\right\}$. Then, choosing $p$ large enough, we have $\|u\|_{L_{d \bar{s}^{2}}^{2}} \leq$ $k\|u\|_{L_{d \tilde{s}^{2}}^{p}}$.

Remark. If $\mu \geq 1$ for all ends, then $d \bar{s}^{2}$ is bounded on all of $\Sigma$, and the lemma holds even for $p=2$. We could argue this way: suppose $u \in L_{d \tilde{s}^{2}}^{2}$. Then

$$
\int_{\Sigma} u^{2} d \bar{A}=\int_{\Sigma} u^{2} \frac{1}{2}|d G|_{d \tilde{s}^{2}}^{2} d \tilde{A} \leq \text { (const) } \int_{\Sigma} u^{2} d \tilde{A} .
$$

Thus $\|u\|_{L_{d \bar{s}^{2}}^{2}} \leq$ (const) $\|u\|_{L_{d \tilde{s}^{2}}^{2}}$. So $L_{d \tilde{s}^{2}}^{2}$ is continuously included in $L_{d \bar{s}^{2}}^{2}$.

We define $\tilde{H}^{1}(\Sigma)=\left\{u \in L_{d \tilde{s}^{2}}^{2} \mid d u \in L_{d \tilde{s}^{2}}^{2}\right\}$, where the derivative

$$
d u=\left(\frac{\partial u}{\partial x_{1}}, \frac{\partial u}{\partial x_{2}}\right)
$$

satisfies

$$
\int_{\Sigma}\left\langle\frac{\partial u}{\partial x_{i}}, \varphi\right\rangle_{d \tilde{s}^{2}} d \tilde{A}=\int_{\Sigma}\left\langle u, \frac{\partial \varphi}{\partial x_{i}}\right\rangle_{d \tilde{s}^{2}} d \tilde{A}
$$

for all test functions $\varphi \in C^{\infty}(\Sigma)$ and all coordinate functions $x_{i}$. The condition that $d u$ must satisfy depends on $d \tilde{s}^{2}$, but it is well known that $\tilde{H}^{1}$ is independent of $d \tilde{s}^{2}$ if $d \tilde{s}^{2}$ is a true metric and not a pseudometric. We define $\bar{H}^{1}(\Sigma)=\left\{u \in L_{d \bar{s}^{2}}^{2} \mid d u \in L_{d \bar{s}^{2}}^{2}\right\}$, where $d u$ satisfies $\int_{\Sigma}\left\langle\partial u / \partial x_{i}, \varphi\right\rangle_{d \bar{s}^{2}} d \bar{A}=$ $\int_{\Sigma}\left\langle u, \partial \varphi / \partial x_{i}\right\rangle_{d \bar{s}^{2}} d \bar{A}$ for all test functions $\varphi \in C^{\infty}(\Sigma)$ and all coordinate functions $x_{i}$. Note that $d \bar{s}^{2}$ is a psuedometric and might not be a true metric even away from the ends $p_{j}$ of the surface, since the secondary Gauss map may have branch points even at finite points on the surface. We define the two norms:

$$
\begin{aligned}
\tilde{\|} u \tilde{\|}^{2} & :=\int_{\Sigma}\left(|\tilde{\nabla} u|_{d \tilde{s}^{2}}^{2}+u^{2}\right) d \tilde{A} \\
\overline{\|} u \overline{\|}^{2} & :=\int_{\Sigma}\left(|\bar{\nabla} u|_{d \bar{s}^{2}}^{2}+u^{2}\right) d \bar{A}=\int_{\Sigma}\left(|\tilde{\nabla} u|_{d \tilde{s}^{2}}^{2}+\frac{1}{2}|d G|_{d \tilde{s}^{2}}^{2} u^{2}\right) d \tilde{A} .
\end{aligned}
$$

Lemma 4.3 $\bar{H}^{1}$ is continuously contained in $\tilde{H}^{1}$. 
Proof. We need to show that there exists a $c>0$ such that $\tilde{\|} \cdot \tilde{\|} \leq c \overline{\|} \cdot \overline{\|}$.

By way of contradiction, suppose that such a $c$ cannot exist. Then there exists a sequence $\left\{u_{n}\right\}_{n=1}^{\infty}$ of functions such that $\tilde{\|} u_{n} \tilde{\|}=1$ and $\overline{\|} u_{n} \overline{\|}<1 / n$. Note the following three facts:

- Any bounded sequence in a Hilbert space has a weakly convergent subsequence (see, for example, $[\mathrm{GT}]$, p. 85). In our case the Hilbert space is $\left(\tilde{H}^{1}, \tilde{\|} \cdot \tilde{\|}\right)$.

- The inclusion of $\tilde{H}^{1}$ into $L_{d \tilde{s}^{2}}^{p}$ is compact for all $p \in[2, \infty)$ (See, for example, $[\mathrm{GT}]$, Theorem 7.22 , or see $[\mathrm{Ad}]$.)

- $\tilde{\|} \cdot \tilde{\|}$ is lower semicontinuous with respect to weak convergence; that is, if $u_{n} \rightarrow u$ weakly, then $\tilde{\|} u \| \leq \liminf _{n \rightarrow \infty} \tilde{\|} u_{n} \tilde{\|}$.

By the first fact, we may assume that $\left\{u_{n}\right\}$ converges weakly in $\tilde{H}^{1}$ to some $u \in \tilde{H}^{1}$. By the second fact, we may assume that $\left\{u_{n}\right\}$ converges strongly in $L_{d \tilde{s}^{2}}^{2}$ to some $v \in L_{d \tilde{s}^{2}}^{2}$. Since $\tilde{H}^{1} \subset L_{d \tilde{s}^{2}}^{2}$ continuously, we have that $u_{n} \rightarrow u \in L_{d \tilde{s}^{2}}^{2}$ weakly. And since the weak limit is unique, we have $u=v$.

By the third fact, we have

$$
\int_{\Sigma}\left(|\tilde{\nabla} u|_{d \tilde{s}^{2}}^{2}+u^{2}\right) d \tilde{A} \leq \liminf _{n \rightarrow \infty} \int_{\Sigma}\left(\left|\tilde{\nabla} u_{n}\right|_{d \tilde{s}^{2}}^{2}+u_{n}^{2}\right) d \tilde{A}
$$

We have strong convergence of $u_{n}$ to $u$ in $L_{d \tilde{s}^{2}}^{2}$, hence $\int_{\Sigma} u_{n}^{2} d \tilde{A} \rightarrow \int_{\Sigma} u^{2} d \tilde{A}$. So we have

$$
\int_{\Sigma}|\tilde{\nabla} u|_{d \tilde{s}^{2}}^{2} d \tilde{A} \leq \liminf _{n \rightarrow \infty} \int_{\Sigma}\left|\tilde{\nabla} u_{n}\right|_{d \tilde{s}^{2}}^{2} d \tilde{A} .
$$

And then since $\int_{\Sigma}\left|\tilde{\nabla} u_{n}\right|_{d \tilde{s}^{2}}^{2} d \tilde{A}=\int_{\Sigma}\left|\bar{\nabla} u_{n}\right|_{d \bar{s}^{2}}^{2} d \bar{A}<1 / n$, we have

$$
\int_{\Sigma}|\tilde{\nabla} u|_{d \tilde{s}^{2}}^{2} d \tilde{A}=0
$$

Therefore $u$ is constant almost everywhere. Since $1-1 / n \leq \int_{\Sigma} u_{n}^{2} d \tilde{A} \leq 1$ we have $\int_{\Sigma} u^{2} d \tilde{A}=1$ (Here again we are using that $u_{n} \rightarrow u$ strongly in $L_{d \tilde{s}^{2}}^{2}$.) Therefore $u$ is equal to a nonzero constant almost everywhere.

By the previous lemma, $L_{d \tilde{s}^{2}}^{p}$ is continuously included in $L_{d \bar{s}^{2}}^{2}$ for $p$ large enough. By the second fact, $\tilde{H}^{1}$ is compactly contained in $L_{d \tilde{s}^{2}}^{p}$, so it follows that $\tilde{H}^{1}$ is compactly contained in $L_{d \bar{s}^{2}}^{2}$. This means that any weakly convergent sequence in $\tilde{H}^{1}$ (which is therefore a bounded sequence in $\tilde{H}^{1}$ ) has a strongly convergent subsequence in $L_{d \bar{s}^{2}}^{2}$. So, since $\int_{\Sigma} u_{n}^{2} d \bar{A} \leq \frac{1}{n}$, we have $\int_{\Sigma} u^{2} d \bar{A}=0$. But $u$ is a nonzero constant, so $0=\int_{\Sigma} u^{2} d \bar{A}=($ const $\neq 0) \cdot \int_{\Sigma} \frac{1}{2}|d G|_{d \tilde{s}^{2}}^{2} d \tilde{A}$. Therefore $|d G|_{d \tilde{s}^{2}}=0$ almost everywhere, and thus $\left|G^{\prime}\right|=0$. Hence

$$
K=-4\left(\frac{\left|G^{\prime}\right|^{2}}{\left|g^{\prime}\right||f|\left(1+|G|^{2}\right)^{2}}\right)^{2}=0
$$


which implies the surface is umbilic, and hence a horosphere. But we assumed the surface is not a horosphere, so this is a contradiction.

Remark. If $\mu \geq 1$ for all ends of a constant mean curvature 1 surface $M$, then $\tilde{H}^{1}=\bar{H}^{1}$. We already know that $\bar{H}^{1}$ is continuously included in $\tilde{H}^{1}$, so to show this it remains only to show that there exists a $c>0$ such that $\overline{\|} \cdot \overline{\|} \leq c \tilde{\|} \cdot \tilde{\|}$. At points where $G$ is not branched we can make a local expression $G=a z+b z^{2}+\cdots$ with $a \neq 0$. We may assume $d \tilde{s}^{2}$ is the Euclidean metric locally, so $|d G|_{d \tilde{s}^{2}}=a$ at the chosen point . At points where $G$ is branched we can make a local expression $\bar{G}=a z^{m}+b z^{m+1}+\cdots$ with $a \neq 0$ and $m \in \mathbb{Z}$, $m \geq 2$. In this case $|d G|_{d \tilde{s}^{2}}=0$ at the chosen point. At each end we can make a local expression $G=z^{\mu}\left(a+b z+c z^{2}+\cdots\right)$ with $a \neq 0$ and $\mu \geq 1$. In this case $|d G|_{d \tilde{s}^{2}}=0$ at the chosen point if $\mu>1$, and $|d G|_{d \tilde{s}^{2}}=a$ at the chosen point if $\mu=1$. In any case $|d G|_{d \tilde{s}^{2}}$ is bounded, and the existence of $c$ follows.

Remark. Since $d \bar{s}^{2}$ is identically zero for the horosphere, the calculations in this section would have no meaning for this example. And as it is the only example for which $d \bar{s}^{2}$ is zero at more than just isolated points, it is natural to exclude it. In any case, the index of the horosphere is easily seen to be 0 (see Section 6).

Lemma 4.4 $\bar{H}^{1}$ is compactly contained in $L_{d \bar{s}^{2}}^{2}$.

Proof. $\bar{H}^{1}$ is continuously contained in $\tilde{H}^{1}$, and $\tilde{H}^{1}$ is compactly contained in $L_{d \tilde{s}^{2}}^{p}$ for any value of $p$, and $L_{d \tilde{s}^{2}}^{p}$ is continuously contained in $L_{d \bar{s}^{2}}^{2}$ if $p$ is large enough. The composition of a continuous map and a compact map and a continuous map is compact.

We remark that this section above and Lemma 5.2 below have an indirect, but close, relationship with the works of Troyanov and others on Riemannian surfaces with conical singularities $[\mathrm{HT}],[\mathrm{T}]$.

5. The relationship between $\operatorname{Ind}(M)$ and eigenvalues of $\bar{L}$. The last lemma in the previous section will lead us to an argument that $\operatorname{Ind}(\Sigma)$ is equal to the number of negative eigenvalues of $\bar{L}$ on $\Sigma$. (We are about to define $\operatorname{Ind}(\Sigma)$ and $\bar{L}$.) First we show that Rayleigh quotient $\mathcal{Q}$ (as defined in the next lemma) is well defined for any smooth function on $\Sigma$. This next lemma will allow us to start the minimization process (i.e. $\mathcal{Q}(u)<\infty$ for some $u$ ) in the proof of the lemma which comes after it.

Before considering the next lemma, we define the relevant Jacobi operators. The original Jacobi operator on $M$ is $L=\triangle-|d G|_{d s^{2}}^{2}=\triangle+2 K$ on $\Sigma$. The Jacobi operator created by pulling back the metric on the sphere via $G$ is $\bar{L}=$ 
$(-1 / K) \Delta-|d G|_{d \bar{s}^{2}}^{2}=\bar{\Delta}-2$ on $\Sigma$. Note that $\bar{L}$ is defined everywhere on $\Sigma$ except at the isolated points where $d \bar{s}^{2}=0$ and possibly at points that represent the ends of $M$. The operator associated to the regular metric $d \tilde{s}^{2}$ is $\tilde{L}=\tilde{\Delta}-|d G|_{d \tilde{s}^{2}}^{2}$, and is defined on all of $\Sigma$, except possibly at points that represent the ends of $M$. We have

$$
\int_{\Sigma} u \tilde{L} u d \tilde{A}=\int_{\Sigma} u L u d A=\int_{\Sigma} u \bar{L} u d \bar{A} .
$$

Since $L, \bar{L}$, and $\tilde{L}$ are not well defined only at isolated points of $\Sigma$, these integrals are well defined.

\section{Lemma 5.1}

$$
\mathcal{Q}(u):=\frac{\int_{\Sigma} u \bar{L} u d \bar{A}}{\int_{\Sigma} u^{2} d \bar{A}}<\infty \text { for all } u \in C^{\infty}(\Sigma)
$$

Proof. Locally at each end, we can normalize $G(z)$ to be $G=$ $z^{\mu}\left(1+a_{1} z+a_{2} z^{2}+\cdots\right)$, where $z$ is contained in a neighborhood $U$ of $z=0$, and $z=0$ represents the end, and $d \tilde{s}^{2}$ is the Euclidean metric on $U$, and $\mu>0$, and $|d G|_{d \tilde{s}^{2}}^{2} \approx 8 \mu^{2}|z|^{2 \mu-2} /\left(\left(1+|z|^{2 \mu}\right)^{2}\right)$. (We showed this in the proof of Lemma 4.2.) Since $\int_{\Sigma} u \tilde{L} u d \tilde{A}=\int_{\Sigma} u \bar{L} u d \bar{A}$, we can show that the numerator of $\mathcal{Q}(u)$ is finite by showing that $\int_{\Sigma} u \tilde{L} u d \tilde{A}$ is finite. To show this, it is sufficient to show that $\int_{U} u \tilde{L} u d \tilde{A}$ is finite at each end, since $u \in C^{\infty}(\Sigma)$, and $d \tilde{s}^{2}$ is nonsingular on the compact $\Sigma$, and $\tilde{L}$ is nonsingular on $\Sigma$ away from the ends.

Since $u \in C^{\infty}(\Sigma)$, we have that $u, u_{x}, u_{y}$ are all bounded on $U$. Since $d \tilde{s}^{2}$ is the Euclidean metric on $U$, we have that $d \tilde{A}=r d r d \theta$ in polar coordinates on $U$. Furthermore, we have $\mu>0$, hence

$$
\left|\int_{U}\left(u_{x}^{2}+u_{y}^{2}-\frac{8 \mu^{2} r^{2 \mu-2} u^{2}}{\left(1+r^{2 \mu}\right)^{2}}\right) r d r d \theta\right|<\infty .
$$

So the numerator of $\mathcal{Q}(u)$ is finite, and therefore $|\mathcal{Q}(u)|<\infty$.

Given any closed region $\Omega \subset \Sigma \backslash\left\{p_{j}\right\}$, we can consider the Dirichlet problem $L u=\lambda u$ and $\bar{L} u=\lambda u$ on $\Omega$ with $\left.u\right|_{\partial \Omega}=0$. In general, $L$ and $\bar{L}$ will have different eigenvalues on $\Omega$; however, supposing that $V$ is some vector space of functions with compact support on $\Omega, \mathcal{Q}(u)<0$ for all $u \in V$ if and only if

$$
\frac{\int_{\Omega} u L u d A}{\int_{\Omega} u^{2} d A}<0
$$

for all $u \in V$. We define $\operatorname{Ind}(\bar{L}, \Omega)$ to be the maximum possible dimension of a subspace of functions in $C_{0}^{\infty}(\Omega)$ on which $\mathcal{Q}(u)<0$. We define $\operatorname{Ind}(L, \Omega)$ to 
be the maximum possible dimension of a subspace of functions in $C_{0}^{\infty}(\Omega)$ on which $\left(\int_{\Omega} u L u d A\right) /\left(\int_{\Omega} u^{2} d A\right)<0$. Thus $\operatorname{Ind}(\bar{L}, \Omega)=\operatorname{Ind}(L, \Omega)$. We consider a sequence of regions $\left\{\Omega_{i}\right\}_{i=1}^{\infty}$ such that $\Omega_{i} \subset \Omega_{i+1}$ and $\bigcup \Omega_{i}=\Sigma \backslash\left\{p_{j}\right\}$. We define $\operatorname{Ind}(\bar{L}, M)=\lim _{i \rightarrow \infty} \operatorname{Ind}\left(\bar{L}, \Omega_{i}\right)$, and we define $\operatorname{Ind}(L, M)=\lim _{i \rightarrow \infty} \operatorname{Ind}\left(L, \Omega_{i}\right)$. It follows that $\operatorname{Ind}(\bar{L}, M)=\operatorname{Ind}(L, M)$. And by the definition given in the second section, $\operatorname{Ind}_{u}(M)=\operatorname{Ind}(L, M)$. Defining $\operatorname{Ind}(\Sigma):=\operatorname{Ind}(\bar{L}, \Sigma)$ to be the maximum possible dimension of a subspace of functions in $C^{\infty}(\Sigma)$ on which $\mathcal{Q}(u)<0$, we have

$$
\operatorname{Ind}_{u}(M) \leq \operatorname{Ind}(\Sigma) .
$$

In order to explicitly compute $\operatorname{Ind}_{u}(M)$ and to show that $\operatorname{Ind}_{u}(M)=\operatorname{Ind}(\Sigma)$, we would like to know that $\operatorname{Ind}(\Sigma)$ equals the number of negative eigenvalues of $\bar{L}$ on $\Sigma$. That this holds (Corollary 5.1) can be concluded from the next lemma. The fact that $\operatorname{Ind}(\Sigma)$ equals the number of negative eigenvalues of $\bar{L}$ on $\Sigma$ is very useful for making explicit estimates of $\operatorname{Ind}(M)$, as we shall see.

Lemma 5.2 We can find weak solutions $u \in \bar{H}^{1}$ of $\bar{L} u=\lambda u$ on $\Sigma$ so that the following hold:

- The set of eigenvalues consists of an infinite sequence

$$
\lambda_{1}<\lambda_{2}<\ldots \rightarrow \infty .
$$

- Each eigenvalue has finite multiplicity and the eigenspaces (of weak solutions) corresponding to distinct eigenvalues are $L_{d \bar{s}^{2}}^{2}$ orthogonal.

- The direct sum of the eigenspaces is dense in $L_{d \bar{s}^{2}}^{2}$ for the $L_{d \bar{s}^{2}}^{2}$ norm.

- Any eigenfunction $u$ of $\lambda_{j}$ is contained in $C^{\infty}\left(\Sigma \backslash\left\{p_{j}\right\}\right)$ and satisfies $\bar{L} u=$ $\lambda_{j} u$ in the classical sense on $\Sigma \backslash\left\{p_{j}\right\}$.

- Any eigenfunction $u$ of $\lambda_{j}$ is contained in $C^{0}(\Sigma)$.

Proof. The Rayleigh-Ritz quotient as defined in Lemma 5.1 is

$$
\mathcal{Q}(u):=\frac{\int_{\Sigma}|d u|_{d \bar{s}^{2}}^{2}-2 u^{2} d \bar{A}}{\int_{\Sigma} u^{2} d \bar{A}}=\frac{\int_{\Sigma}|d u|_{d \tilde{s}^{2}}^{2}-|d G|_{d \tilde{s}^{2}}^{2} u^{2} d \tilde{A}}{\int_{\Sigma} \frac{1}{2}|d G|_{d \tilde{s}^{2}}^{2} u^{2} d \tilde{A}}, \quad u \in \bar{H}^{1} .
$$

The denominator is the $L_{d \bar{s}^{2}}^{2}$ norm.

The proof of the first three items follows by standard variational arguments, such as in the arguments on pages 55-59 of [Be]. The only difference between the proof of the lemma above and the proof in [Be] is that elliptic regularity is used there to show that the eigenfunctions corresponding to the eigenvalues are classical solutions of the eigenvalue problem (on all of $\Sigma$ ). In our case we only conclude that we have weak solutions to the eigenvalue problem. However, we can simply ignore the arguments where elliptic regularity is used, and the remaining arguments in [Be] are sufficient to prove the first three items in the lemma above, so we shall not repeat the arguments here. 
We remark that in order to apply these standard variational arguments, it is crucial that we know that $\bar{H}^{1}$ is compactly included in $L_{d \bar{s}^{2}}^{2}$. This is why we were focusing on proving Lemma 4.4 in the previous section.

We now turn to proving the last two items in the lemma. Suppose $u$ is a weak solution of $\bar{L} u=\lambda_{j} u$, so $\tilde{\Delta} u=q u$ in the weak sense, where $q=\left(1+\lambda_{j} / 2\right)|d G|_{d \tilde{s}^{2}}^{2}$. Since $q \in C^{\infty}\left(\Sigma \backslash\left\{p_{j}\right\}\right)$, it follows from elliptic regularity ([GT], Corollary 8.11) that $u \in C^{\infty}\left(\Sigma \backslash\left\{p_{j}\right\}\right)$ and satisfies $\bar{L} u=\lambda_{j} u$ in the classical sense on $\Sigma \backslash\left\{p_{j}\right\}$.

Consider a small neighborhood $U_{j} \subset \Sigma$ of the point $p_{j}$ representing an end. If $\mu_{j} \geq 1$ at $p_{j}$, then $q \in C^{0}\left(U_{j}\right)$, and therefore any eigenfunction $u$ is contained in $C^{0}\left(U_{j}\right)$ ([GT], Theorem 8.8 and Corollary 7.11). If $\mu_{j}<1$ at $p_{j}$, we will see in the next three paragraphs that $u$ is still contained in $C^{0}\left(U_{j}\right)$.

To show $u \in C^{0}(\Sigma)$, we only need to show $u \in C^{0}\left(U_{j}\right)$, since $u$ is $C^{\infty}$ away from the $p_{j}$. First we state Theorem 17.1.1 from $[\mathrm{H}]$. Consider a linear operator of order $m, P(x, D)=\sum_{|\alpha| \leq m} a_{\alpha}(x) D^{\alpha}$ in an open set $X \subset \mathbb{R}^{n}$. We may assume $X=U_{j}$, since we may choose $d \tilde{s}^{2}$ to be the standard Euclidean metric on $U_{j}$. In our case $P(x, D)=\tilde{\Delta}, m=n=2$. Suppose that $P_{m}(0, D)=\sum_{|\alpha|=m} a_{\alpha}(0) D^{\alpha}$ is elliptic. This is certainly true for $\tilde{\Delta}$. Suppose also that $a_{\alpha}$ in continuous when $|\alpha|=m$, and that for some $r \in(1, \infty), a_{\alpha} \in L_{\mathrm{loc}, d \tilde{s}^{2}}^{n /(m-|\alpha|)}\left(U_{j}\right)$ if $m-|\alpha|<n / r$, and $a_{\alpha} \in L_{\mathrm{loc}, d \tilde{s}^{2}}^{r+\varepsilon}\left(U_{j}\right)$ for some $\varepsilon>0$ if $m-|\alpha|=n / r$, and $a_{\alpha} \in L_{\text {loc, }, d \tilde{s}^{2}}^{r}\left(U_{j}\right)$ if $m-|\alpha|>n / r$. In our case all of the coefficients are constant, so these conditions will hold. The theorem says that if all these conditions are satisfied and $U_{j}$ is a sufficiently small neighborhood of $p_{j}$, then there is a linear operator $E$ in $L_{d \tilde{s}^{2}}^{r}\left(U_{j}\right)$ such that

- $f \in L_{d \tilde{s}^{2}}^{r}\left(U_{j}\right) \rightarrow D^{\alpha} E f \in L_{d \tilde{s}^{2}}^{s}\left(U_{j}\right)$ is continuous if $r \leq s \leq \infty$ and

$$
\frac{1}{s} \geq \frac{1}{r}-\left(\frac{m-|\alpha|}{n}\right)
$$

with strict inequality if $s=\infty$,

- $P(x, D) E f=f, f \in L_{d \tilde{s}^{2}}^{r}\left(U_{j}\right)$,

- $E P(x, D) \mathrm{v}=\mathrm{v}$ if $\mathrm{v} \in C_{0}^{\infty}\left(U_{j}\right)$.

In our case we will have $E f=u$ and $f=q u$. We will choose $1<r=s \approx 1$.

We now show that $q u \in L_{d \tilde{s}^{2}}^{r}\left(U_{j}\right)$ if $r$ is sufficiently close to 1 . Since $u \in \bar{H}^{1}$, Lemma 4.3 implies that $u \in \tilde{H}^{1}$. And since $\tilde{H}^{1}$ is compactly contained in $L_{d \tilde{s}^{2}}^{p}$ for all $p \geq 2$, we have that $\int_{U_{j}} u^{p} d \tilde{A}$ is finite for all $p \geq 2$. As we saw in the proof of Lemma $4.2, \int_{U_{j}} q^{t} d \tilde{A}$ is finite if $t(t>1)$ is sufficiently close to 1 . Choose such a $t$ sufficiently close to 1 , and choose $p$ sufficiently large so that $1 / p+1 / t<1$. Define $r>1, r \approx 1$ by $1 / r=1 / p+1 / t$. By the Hölder inequality,

$$
\left(\int_{U_{j}}(q u)^{r} d \tilde{A}\right)^{1 / r} \leq\left(\int_{U_{j}} q^{t} d \tilde{A}\right)^{1 / t}\left(\int_{U_{j}} u^{p} d \tilde{A}\right)^{1 / p}<\infty .
$$


So we have that $q u \in L_{d \tilde{s}^{2}}^{r}\left(U_{j}\right)$, and, by the theorem from [H] stated above, we have that the map $f \rightarrow D^{\alpha} E f$ from $L_{d \tilde{s}^{2}}^{r}\left(U_{j}\right)$ to $L_{d \tilde{s}^{2}}^{s}\left(U_{j}\right)$ is continuous when $|\alpha| \leq 2$ and $s=r$. Therefore, letting $E f=u$ and $f=q u$, we have $\left\|D^{\alpha} u\right\|_{L_{d \bar{s}^{2}\left(U_{j}\right)}^{s=r}} \leq c\|q u\|_{L_{d \tilde{s}^{2}}^{r}\left(U_{j}\right)}<\infty$ when $|\alpha| \leq 2$. This implies that $u$ is contained in the Sobolev space $W^{2, r}\left(U_{j}\right)$. Then, since $u \in W^{2, r}\left(U_{j}\right)$, we have $u \in C^{0}\left(U_{j}\right)$ (see, for example, [GT], Corollary 7.11).

Corollary $5.1 \operatorname{Ind}(\Sigma)=$ the number of negative eigenvalues (counted with multiplicity) of $\bar{L}$ on $\Sigma$.

This corollary follows immediately from the variational characterization of the eigenvalues (for example, see [Be], p61). The $k^{\prime}$ th eigenvalue $\lambda_{k}$ is characterized by

$$
\lambda_{k}=\inf _{V_{k}} \sup \left\{Q(u) \mid u \in V_{k}, u \neq 0\right\},
$$

where $V_{k}$ runs through all $k$ dimensional subspaces of $\bar{H}^{1}$.

Lemma 5.3 $\operatorname{Ind}_{u}(M)=\operatorname{Ind}(\Sigma)$, and either $\operatorname{Ind}(M)=\operatorname{Ind}(\Sigma)$ or $\operatorname{Ind}(M)=$ $\operatorname{Ind}(\Sigma)-1$

Proof. Let $f_{1}, \ldots, f_{\operatorname{Ind}(\Sigma)}$ be the eigenfunctions of $\bar{L}$ on $\Sigma$ with negative eigenvalues. Since $f_{i} \in \bar{H}^{1}$, we know that

$$
\int_{B_{\varepsilon}\left(p_{j}\right)}\left|f_{i}\right|^{2} d \bar{A} \rightarrow 0 \quad \text { and } \quad \int_{B_{\varepsilon}\left(p_{j}\right)}\left|\bar{\nabla} f_{i}\right|^{2} d \bar{A} \rightarrow 0 \quad \text { as } \varepsilon \rightarrow 0
$$

where $B_{\varepsilon}\left(p_{j}\right)$ is a ball about the end $p_{j} \in \Sigma$ with radius $\varepsilon$ with respect to the $d \tilde{s}^{2}$ metric (these follow from the Lebesque dominated convergence theorem). By the previous lemma, we have $f_{i} \in C^{0}(\Sigma)$, and thus $\left|f_{i}\right| \leq c$, a constant. Using these facts, we can then follow, with only slight modification, the argument in FischerColbrie's proof $[\mathrm{FC}]$. For the sake of completeness, we include the argument here.

In a neighborhood of a point $p_{j}$ representing an end, choose a local complex coordinate $z$ centered at $p_{j}$. For some small $\varepsilon>0$, define a function

$$
\eta_{j}(z)= \begin{cases}0 & \text { if }|z|<\varepsilon^{2}, \\ 1 & \text { if }|z|>\varepsilon, \text { and } \\ \frac{\log \left(\frac{|z|}{\varepsilon^{2}}\right)}{\log \left(\frac{1}{\varepsilon}\right)} & \text { if } \varepsilon^{2} \leq|z| \leq \varepsilon .\end{cases}
$$


Let $\eta=\eta_{j}$ in an $\varepsilon$ ball about each $p_{j}$, and let $\eta=1$ elsewhere. One can check that

$$
\int_{\Sigma}|\bar{\nabla} \eta|^{2} d \bar{A}=\int_{\Sigma}|\tilde{\nabla} \eta|^{2} d \tilde{A} \leq \frac{\hat{c}}{\log \left(\frac{1}{\varepsilon}\right)}
$$

for some constant $\hat{c}$, by noting that $d \tilde{s}^{2} \approx|d z|^{2}$. Therefore $\int_{\Sigma}|\bar{\nabla} \eta|^{2} d \bar{A} \rightarrow 0$ as $\varepsilon \rightarrow 0$.

Let $g_{i}=\eta f_{i}$, then

$$
\int_{\Sigma}\left(g_{i}-f_{i}\right)^{2} d \bar{A}=\int_{\Sigma}(1-\eta)^{2} f_{i}^{2} d \bar{A} \leq \sum_{p_{j}} \int_{B_{\varepsilon}\left(p_{j}\right)}\left|f_{i}\right|^{2} d \bar{A} \rightarrow 0
$$

as $\varepsilon \rightarrow 0$, so $\int_{\Sigma}\left(g_{i}-f_{i}\right)^{2} d \bar{A} \rightarrow 0$ as $\varepsilon \rightarrow 0$. Also,

$$
\begin{aligned}
\int_{\Sigma}\left|\bar{\nabla}\left(g_{i}-f_{i}\right)\right|^{2} d \bar{A} & =\int_{\Sigma}\left|\bar{\nabla}\left((1-\eta) f_{i}\right)\right|^{2} d \bar{A} \\
& \leq 2 \int_{\Sigma}\left[|\bar{\nabla} \eta|^{2} f_{i}^{2}+(1-\eta)^{2}\left|\bar{\nabla} f_{i}\right|^{2}\right] d \bar{A} \\
& \leq 2 c^{2} \int_{\Sigma}|\bar{\nabla} \eta|^{2} d \bar{A}+\sum_{p_{j}} \int_{B_{\varepsilon}\left(p_{j}\right)}\left|\bar{\nabla} f_{i}\right|^{2} d \bar{A}
\end{aligned}
$$

since $\left|f_{i}\right| \leq c$ and $0 \leq(1-\eta)^{2} \leq 1$. Each of the integrals in the sum on the right hand side converge to 0 as $\varepsilon \rightarrow 0$. Hence we have that $\overline{\|} f_{i}-g_{i} \overline{\|}^{2} \rightarrow 0$ as $\varepsilon \rightarrow 0$. By continuity of $\mathcal{Q}$ with respect to the $\bar{H}^{1}$ norm, we have that $\mathcal{Q}$ is negative definite on the span of $\left\{g_{i}\right\}_{i=1}^{\operatorname{Ind}(\Sigma)}$ in $\bar{H}^{1}$ for sufficiently small $\varepsilon$. Therefore $\operatorname{Ind}_{u}(M) \geq$ $\operatorname{Ind}(\Sigma)$, and hence the first part of the lemma follows.

To prove the second part of the lemma, suppose that $V \subset \bar{H}^{1}$ is a vector space of dimension $\operatorname{Ind}_{u}(M)$ on which $\mathcal{Q}<0$. If $V$ is perpendicular to the constant functions with respect to the $L_{d \bar{s}^{2}}^{2}$ inner product, then all of the functions in $V$ are volume preserving, and we have $\operatorname{Ind}(M)=\operatorname{Ind}(\Sigma)$.

If $V$ is not perpendicular to the constant functions, then the perpendicular projection of the constant function 1 to $V$ is a function $\varphi_{1} \neq 0, \varphi_{1} \in V$. We may extend $\varphi_{1}$ to an orthogonal basis $\left\{\varphi_{1}, \varphi_{2}, \ldots, \varphi_{\operatorname{Ind}_{u}(M)}\right\}$ of $V$. Since $\varphi_{2}, \ldots, \varphi_{\operatorname{Ind}_{u}(M)}$ are all perpendicular to $\varphi_{1}$ with respect to the $L_{d \bar{s}^{2}}^{2}$ inner product in $V$, it follows easily that $\varphi_{2}, \ldots, \varphi_{\operatorname{Ind}_{u}(M)}$ are all perpendicular to the constant function 1 in $L_{d \bar{s}^{2}}^{2}$. Thus a subspace of $V$ of $\operatorname{dimension} \operatorname{Ind}_{u}(M)-1$ is perpendicular to the constant functions, so we have constructed a space of volume preserving functions of dimension $\operatorname{Ind}_{u}(M)-1$ on which $\mathcal{Q}<0$, and thus $\operatorname{Ind}(M) \geq \operatorname{Ind}(\Sigma)-1$.

We have the following corollary, which is a result of do Carmo and Silveira ([CS]). The advantage of our proof of this corollary is that our method will allow us to make specific estimates of the index, whereas the method in [CS] would not allow this. 
Corollary 5.2 If a constant mean curvature 1 surface in $\mathbb{H}^{3}$ has finite total curvature, then it has finite index.

Proof. Since the surface has finite total curvature, it has a conformal bijection to $\Sigma \backslash\left\{p_{j}\right\}$. By Lemma $5.2, \bar{L}$ has a finite number of negative eigenvalues. Then, by Corollary $5.1, \operatorname{Ind}(\Sigma)$ is finite. Hence, by Lemma $5.3, \operatorname{Ind}(M)$ is finite.

6. Examples. We now compute the index of several examples, showing how the results of the previous section can be applied. For the sake of completeness, we compute the already known index of the horosphere, before continuing on to new results about index of certain surfaces.

Horosphere: For the horosphere, we can choose $\Sigma=\mathbb{C} \cup\{\infty\}$ and $\Sigma \backslash\left\{p_{j}\right\}=$ $\mathbb{C}$ and $f=1$ and $g=1$ and $c=1$ and $z_{0}=0$ in Lemma 3.2. Writing $F$ as

$$
F=\left(\begin{array}{cc}
A & B \\
C & D
\end{array}\right)
$$

we have

$$
\begin{aligned}
\left(\begin{array}{ll}
A^{\prime} & B^{\prime} \\
C^{\prime} & D^{\prime}
\end{array}\right) & =\left(\begin{array}{cc}
A & B \\
C & D
\end{array}\right)\left(\begin{array}{cc}
1 & -1 \\
1 & -1
\end{array}\right) \rightarrow\left(\begin{array}{cc}
A & B \\
C & D
\end{array}\right) \\
& =\left(\begin{array}{ll}
A_{0} & B_{0} \\
C_{0} & D_{0}
\end{array}\right) e^{\left(\begin{array}{ll}
1 & -1 \\
1 & -1
\end{array}\right) z}
\end{aligned}
$$

and since $\left.F\right|_{z_{0}=0}=$ id, we have $A_{0}=D_{0}=1$ and $B_{0}=C_{0}=0$, therefore

$$
\begin{aligned}
F & =\left(\begin{array}{ll}
A & B \\
C & D
\end{array}\right) \\
& =\left(\begin{array}{ll}
1 & 0 \\
0 & 1
\end{array}\right)+\left(\begin{array}{ll}
1 & -1 \\
1 & -1
\end{array}\right) z+\left\{\left(\begin{array}{ll}
1 & -1 \\
1 & -1
\end{array}\right)^{2}=\left(\begin{array}{ll}
0 & 0 \\
0 & 0
\end{array}\right)\right\} \frac{z^{2}}{2 !}+\cdots,
\end{aligned}
$$

and so

$$
F=\left(\begin{array}{cc}
1+z & -z \\
z & 1-z
\end{array}\right)
$$

Thus $G=1$, and it follows that the curvature $K=0$, and so the second variational formula given in the first section becomes

$$
\left.\frac{d^{2} A}{d t^{2}}\right|_{t=0}=\int_{M}|\nabla u|^{2} d A \geq 0 .
$$

This is nonnegative for all functions $u$, hence the horosphere is stable.

Silveira $([\mathrm{Si}])$ showed that the only complete stable noncompact constant mean curvature 1 surface in $\mathbb{H}^{3}$ is the horosphere. 
Enneper cousin. For the Enneper cousin, we can choose $\Sigma=\mathbb{C} \cup\{\infty\}$ and $\Sigma \backslash\left\{p_{j}\right\}=\mathbb{C}$ and $f=1$ and $g=z$ and $c=1$ and $z_{0}=0$ in Lemma 3.2. Solving the equation

$$
d F=F\left(\begin{array}{cc}
z & -z^{2} \\
1 & -z
\end{array}\right) d z
$$

we find that

$$
F=\left(\begin{array}{cc}
\cosh (z) & \sinh (z)-z \cosh (z) \\
\sinh (z) & \cosh (z)-z \sinh (z)
\end{array}\right) .
$$

Therefore $G=d(\cosh (z)) / d(\sinh (z))=\tanh (z)$.

Following the Weierstrass representation as formulated in [By], we have a constant mean curvature 1 surface given by $F \bar{F}^{t}$ with secondary Gauss map $g=z$. (Note that, since we are using $F$ instead of $F^{-1}$ to make the surface, the function $g$ is now the secondary Gauss map, not the hyperbolic Gauss map.) In this case the secondary Gauss map is actually single valued, since the surface is simply connected. By Lemma 4.1, the second variation is determined by $\Sigma=\mathbb{C} \cup\{\infty\}$ and $g=z$. For this $\Sigma$ and $g$, the unconstrained index is $\operatorname{Ind}_{u}(M)$ $=1$. This can be seen from Theorem 4.6 of [N1], or from Proposition 6.1 below. It follows $\operatorname{Ind}(M)$ is either 0 or 1 . But the Enneper cousin cannot be stable, since the horosphere is the only stable example ([Si]), hence $\operatorname{Ind}(M)=1$.

We can also consider Enneper cousins with winding order $2 k+1$ at the end, $k \in \mathbb{N}$. In this case $g$ becomes $g=z^{k}$, and the other objects $\Sigma=\mathbb{C} \cup\{\infty\}$ and $\Sigma \backslash\left\{p_{j}\right\}=\mathbb{C}$ and $f=1$ and $c=1$ and $z_{0}=0$ remain unchanged. Now, by [N1] or Proposition 6.1 below, $\operatorname{Ind}_{u}(M)=2 k-1$. Hence, by Lemma 5.3, the Enneper cousins with winding order $2 k+1$ have constrained index $\operatorname{Ind}(M)$ either $2 k-1$ or $2 k-2$.

Following the Weierstrass representation as formulated in Lemma 3.2 of this paper, we have a constant mean curvature 1 surface given by $F^{-1} \bar{F}^{-1}$, and this produces the "dual" Enneper cousin that is described in [RUY]. The dual Enneper cousin has secondary Gauss map $G=\tanh (z)$ and hence has infinite total curvature. By [CS], it must therefore have infinite index. (See Figure 1.)

Catenoid cousin. The catenoid cousin has $\Sigma \backslash\left\{p_{j}\right\}=\mathbb{C} \backslash\{0\}$, and has secondary Gauss map $G=z^{\mu}$, where $\mu \neq 0, \pm 1$ is real. We can assume without loss of generality that $\mu>0$. The surface is embedded if $\mu<1$ and not embedded if $\mu>1$. (This is shown in [UY1]. It was originally shown in [By], but the parameter $\mu$ is formulated differently in [By]. We use the same $\mu$ as in the [UY1] formulation. Figures of the catenoid cousins can be found in [UY1].)

We will show that the embedded catenoid cousins have index 1 , and that the non-embedded catenoid cousins have index at least 2, and that the index gets arbitrarily large as $\mu$ gets arbitrarily large. To prove this, we first prove the following proposition. This proposition is proven in [N1] in the case that $\mu$ is an integer. The proof when $\mu$ is not an integer is essentially the same. We include the proof here for the sake of completeness. 
Figure 1: Half of a "dual" Enneper cousin in the Poincaré model. The entire surface consists of the piece shown union its reflection across the plane containing the boundary curve of this piece. This surface has infinite total curvature, and therefore has infinite index.

Proposition 6.1 Let $\mu$ be a positive real. The complete set of eigenvalues for the Laplacian on the plane with the pull back metric from the sphere via the map $G=z^{\mu}$ is

$$
\lambda_{p, q}=\left(p+\frac{q}{\mu}\right)\left(1+p+\frac{q}{\mu}\right), \quad p, q=0,1,2, \ldots
$$

The multiplicity of $\lambda_{p, q}$ is 2 if $q>0$ and is 1 if $q=0$.

Proof. We are considering the problem $\bar{\Delta} u=\lambda u$, where $\bar{\Delta}$ is the Laplacian obtained from pulling back the standard metric on $S^{2}$ via the map $G=z^{\mu}$. In polar coordinates this equation becomes

$$
\frac{\partial^{2} u}{\partial r^{2}}+\frac{1}{r} \frac{\partial u}{\partial r}+\frac{1}{r^{2}} \frac{\partial^{2} u}{\partial \theta^{2}}=-\lambda \frac{4 \mu^{2} r^{2 \mu-2}}{\left(r^{2 \mu}+1\right)^{2}} u
$$


For a real number $\alpha$ and a nonnegative integer $i$, we define $(\alpha)_{i}$ to be $(\alpha)_{i}=$ $\alpha(\alpha+1) \ldots(\alpha+i-1)$ if $i>0$ and $(\alpha)_{i}=1$ if $i=0$. We then define a real analytic hypergeometric function $F(a, b, c, x)$ where $c$ is not a nonpositive integer. This function $F(a, b, c, x)$ is defined for $-1<x<1$.

$$
F(a, b, c, x):=\sum_{i=0}^{\infty} \frac{(a)_{i}(b)_{i}}{i !(c)_{i}} x^{i}
$$

$F(a, b, c, x)$ satisfies the hypergeometric differential equation

$$
x(1-x) \frac{d^{2} y}{d x^{2}}+(c-(a+b+1) x) \frac{d y}{d x}-a b y=0 .
$$

For nonnegative integers $p$ and $q$,

$$
F\left(p+2 \frac{q}{\mu}+1,-p, \frac{q}{\mu}+1, \frac{1}{2}(1-t)\right)
$$

is a polynomial of degree $p$. We set

$$
\varphi_{p, q}(t)=\left(1-t^{2}\right)^{q /(2 \mu)} F\left(p+2 \frac{q}{\mu}+1,-p, \frac{q}{\mu}+1, \frac{1}{2}(1-t)\right), \quad-1<t<1,
$$

and

$$
v_{p, q}(r)=\varphi_{p, q}\left(\frac{r^{2 \mu}-1}{r^{2 \mu}+1}\right), \quad 0<r<\infty .
$$

We can check that, for $\lambda=(p+q / \mu)(1+p+q / \mu), \varphi_{p, q}(t)$ satisfies the ordinary differential equation

$$
\left(1-t^{2}\right) \frac{\partial^{2} \varphi}{\partial t^{2}}-2 t \frac{\partial \varphi}{\partial t}+\left(\lambda-\left(\frac{q}{\mu}\right)^{2} \frac{1}{1-t^{2}}\right) \varphi=0
$$

and $v_{p, q}(r)$ satisfies the ordinary differential equation

$$
\frac{\partial^{2} v}{\partial r^{2}}+\frac{1}{r} \frac{\partial v}{\partial r}+\left(\lambda \frac{4 \mu^{2} r^{2 \mu-2}}{\left(r^{2 \mu}+1\right)^{2}}-\frac{q^{2}}{r^{2}}\right) v=0 .
$$

We can then check that $v_{p, q}(r) \cos (q \theta)$ and $v_{p, q}(r) \sin (q \theta)$ are eigenfunctions of the Laplacian with eigenvalue $\lambda=(p+q / \mu)(1+p+q / \mu)$.

Finally, we need to check that, for nonnegative $p$ and $q$, the eigenfunctions above form a complete orthogonal system in the $L_{d \bar{s}^{2}}^{2}$ norm. This follows by elementary arguments.

The following theorem follows immediately from Proposition 6.1 and Lemma 5.3 , and from Silveira's result that the horosphere is the only stable complete constant mean curvature 1 surface in $\mathbb{H}^{3}[\mathrm{Si}]$. 
Theorem 6.1 The index of any embedded catenoid cousin is exactly 1, and the index of any nonembedded catenoid cousin is at least 2 . Let $[\mu]$ be the greatest integer that is strictly less than $\mu$, then the index of the catenoid with value $\mu$ is either $2[\mu]+1$ or $2[\mu]$. Thus, for any positive number $N$ there exists a catenoid cousin with index greater than $N$.

Remark. It is clear from the proposition above that when $\mu \notin \mathbb{Z}$, the nullity (nullity $:=$ the dimension of the eigenspace corresponding to the eigenvalue 0 ) of the catenoid cousins is 1 (i.e. this is the case when $p=1, q=0$ ), and that when $\mu \in \mathbb{Z}$, the nullity is $3(p=1, q=0$; or $p=0, q=\mu)$. And the unconstrained index $\operatorname{Ind}_{u}(M)$ changes only as $\mu$ passes through an integer, when two eigenvalues pass through 0 . Furthermore, this illustrates another difference from the case of minimal surfaces in $\mathbb{R}^{3}$, where the nullity is always at least 3 , since the set of translations make bounded normal Jacobi fields on a minimal surface ([N1], $[\mathrm{MR}],[\mathrm{EK}])$.

There are some other examples where we can compute the index explicitly, via the proposition above, which we will now describe.

Example 7.4 of [UY1] has a Weierstrass representation with $G=z^{m}, 3 \leq$ $m \in \mathbb{Z}$ on $\Sigma \backslash\left\{p_{j}\right\}=\mathbb{C} \backslash\{0\}$. It follows immediately from Proposition 6.1 and Lemma 5.3 that the index $\operatorname{Ind}(M)$ of this example is either $2 m-1$ or $2 m-2$.

Another example is given in Theorem 6.2 of [UY1]. It has a Weierstrass representation with $G=a z^{\ell}+b$ and Hopf differential $Q=a c \ell z^{-2}(d z)^{2}$ on $\Sigma \backslash\left\{p_{j}\right\}=\mathbb{C} \backslash\{0\}$, where $\ell \in \mathbb{Z}, \ell \neq 0$ and $a, b, c \in \mathbb{C}, a \neq 0, c \neq 0$, and $\ell^{2}+4 a c \ell=m^{2}$ for some positive integer $m$.

In the case that $b=0$, we can simply rewrite $a^{1 / \ell} z$ as $z$, and then $\Sigma \backslash\left\{p_{j}\right\}$ is unchanged and $G$ becomes $G=z^{\ell}$. Hence, when $b=0, \operatorname{Ind}(M)$ is either $2 \ell-1$ or $2 \ell-2$, by Proposition 6.1 and Lemma 5.3 .

In the case that $\ell=1$, then we can make the transformation of the complex plane $z \rightarrow(z-b) / a$. Then $\Sigma$ is still $\mathbb{C} \cup\{\infty\}$, and $G$ becomes $G=z^{1}$. Hence by Proposition 6.1 and Lemma 5.3, $\operatorname{Ind}(M)$ is either 0 or 1. By [Si] these surfaces cannot be stable, hence $\operatorname{Ind}(M)=1$. There are many different examples of this type with $\ell=1$ : for example, $\ell=1, a=1, c=2, m=3, b=0$ or $\ell=1, a=\frac{3}{4}$, $c=1, m=2, b=0$, and infinitely many others.

Remark. This last example with $\ell=1$ illustrates another difference between minimal surfaces in $\mathbb{R}^{3}$ and constant mean curvature surfaces in $\mathbb{H}^{3}$ : While the only complete minimal surfaces in $\mathbb{R}^{3}$ with index 1 are the catenoid and Enneper's surface $([\mathrm{FC}],[\mathrm{Cho}])$, the embedded catenoid cousins and the Enneper cousins are not the only constant mean curvature 1 surfaces in $\mathbb{H}^{3}$ with index 1 .

7. Lower bounds for Ind $(M)$. Choe [Cho] proved some general results about lower bounds for the index of minimal surfaces in $\mathbb{R}^{3}$. In this section, we will 
apply the same method to constant mean curvature 1 surfaces in $\mathbb{H}^{3}$. The results in Section 5, particularly Lemma 5.3, are crucial to getting the method to work in our situation.

Let $\varphi$ be a Killing vector field in $\mathbb{H}^{3}$ generated by either a hyperbolic rotation or a hyperbolic translation. For both a hyperbolic rotation and a hyperbolic translation there are two fixed points on the sphere at infinity, and we shall call these two points the points in the sphere at infinity fixed by $\varphi$. (For example, a Euclidean rotation about the $x_{3}$-axis of the upperhalf space model for $\mathbb{H}^{3}$ is a hyperbolic rotation, and a Euclidean dilation centered at the point $x_{1}=x_{2}=$ $x_{3}=0$ of the upperhalf space model is a hyperbolic translation. Both of these isometries of $\mathbb{H}^{3}$ fix the two points $x_{1}=x_{2}=x_{3}=0$ and $x_{1}=x_{2}=x_{3}=\infty$ in the sphere at infinity.)

Let $M$ be a constant mean curvature 1 surface in $\mathbb{H}^{3}$ with finite total curvature. The Killing vector field $\varphi$ can be decomposed into tangent and normal parts on $M$, that is, $\varphi=\varphi^{T}+\varphi^{\perp}$, where $\varphi^{T} \in T(M)$ and $\varphi^{\perp} \in N(M)$, and $N(M)$ is the normal vector bundle of $M$. Choosing a unit normal $\vec{N}$ on $M$, it can be checked by a direct computation (see, for example, Lemma 1 of [Cho] or Proposition 2.12 of [BCE]) that the normal projection $\varphi^{\perp}=u \vec{N}$ of a Killing vector field $\varphi$ on $M$ is a Jacobi field (i.e. $\Delta u+2 K u=0$ ).

Definition 7.1 Let $H(M, \varphi)$ be the set of all points on $M$ where $\varphi^{\perp}=$ 0 . We call $H(M, \varphi)$ the horizon of $M$ with respect to $\varphi$. Each component of $M \backslash H(M, \varphi)$ is called a visible set of $M$. The number of visible sets of $M \backslash$ $H(M, \varphi)$ is called the vision number $v(M, \varphi)$ of $M$ with respect to $\varphi$. The number of visible sets of $M \backslash H(M, \varphi)$ which are either bounded or whose closure intersects the sphere at infinity only at one or both of the points fixed by $\varphi$ is called the adjusted vision number $\tilde{v}(M, \varphi)$ of $M$ with respect to $\varphi$.

Note that $\tilde{v}(M, \varphi) \leq v(M, \varphi)$.

Theorem 7.1 Let $M$ be a constant mean curvature 1 surface in $\mathbb{H}^{3}$ of finite total curvature with regular ends. Then for any choice of $\varphi$,

$$
\operatorname{Ind}(M) \geq \begin{cases}\tilde{v}(M, \varphi)-1 & \text { if } \tilde{v}(M, \varphi) \neq v(M, \varphi), \text { and } \\ \tilde{v}(M, \varphi)-2 & \text { if } \quad \tilde{v}(M, \varphi)=v(M, \varphi)\end{cases}
$$

Proof. First we show that on any visible set which is counted in $\tilde{v}(M, \varphi)$, $u \vec{N}$ is bounded. (This is not true for the visible sets which are not counted in $\tilde{v}(M, \varphi)$.) For this, we need to use that we have regular finite total curvature ends. Note that the definition of a regular end is an end for which the hyperbolic Gauss map $G$ extends holomorphically across $p_{j}$ [UY1]. An end with finite total curvature is regular if and only if $\operatorname{ord}_{p_{j}}(Q) \geq-2[\mathrm{By}]$. For these types of ends, assuming that the end approaches the origin in the upper-half-space model, we have the following asymptotic behavior:

$$
\left(\operatorname{Re}\left(z^{m}\right), \operatorname{Im}\left(z^{m}\right), c|z|^{\mu+m}\left(1+\mathcal{O}\left(|z|^{\min (1,2 \mu)}\right)\right)\right)
$$


where $z$ is a local coordinate at the end, $z=0$ is the point representing the end, and $c$ is a positive constant. $\mathcal{O}(1,2 \mu)=\mathcal{O}\left(|z|^{\min (1,2 \mu)}\right)$ denotes any real valued function $f(z) \operatorname{such}$ that $\lim \sup _{z \rightarrow 0}|f| /\left(|z|^{\min (1,2 \mu)}\right)$ is bounded. (See the appendix for a proof of this asymptotic behavior.) Note that the unit normal $\vec{N}$ is of the form

$$
\begin{aligned}
\vec{N}=\frac{c x^{\mu+m}}{\sqrt{m^{2}+c^{2}(\mu+m)^{2} x^{2 \mu}+\mathcal{O}(1,2 \mu)}} & \\
& \times\left(-c(\mu+m) x^{\mu}(1+\mathcal{O}(1,2 \mu)), x^{\mu} \mathcal{O}(1,2 \mu), m\right)
\end{aligned}
$$

over a point $z=x>0, x \in \mathbb{R}$.

We now consider three cases for the Killing vector field $\varphi$ :

- Suppose $\varphi$ is made by an isometry which is either a hyperbolic rotation or a hyperbolic translation, and suppose that the origin $x_{1}=x_{2}=x_{3}=0$ is not one of the two points in the sphere at infinity fixed by $\varphi$. In this case we may consider that $\varphi \approx(\overrightarrow{1,0,0})$ near the origin. Thus, when $z=x>0$, we have

$$
\langle\varphi, \vec{N}\rangle_{\mathbb{H}^{3}} \approx \frac{-(\mu+m)(1+\mathcal{O}(1,2 \mu))}{x^{m} \sqrt{m^{2}+c^{2}(\mu+m)^{2} x^{2 \mu}+\mathcal{O}(1,2 \mu)}},
$$

and this will diverge to $\infty$ as $x \rightarrow 0$. Thus for a $\varphi$ of this type, the normal Jacobi vector field $\varphi^{\perp}=\langle\varphi, \vec{N}\rangle \vec{N}$ is not bounded. (As a simple example, one can easily compute $\langle\varphi, \vec{N}\rangle$ explicitly for a horosphere.)

- Suppose $\varphi$ is made by the isometry which is a dilation centered at the origin. In this case $\varphi=\left(\overrightarrow{x_{1}, x_{2}, x_{3}}\right)$ at $\left(x_{1}, x_{2}, x_{3}\right)$. Thus, when $z=x>0$, we have

$$
\langle\varphi, \vec{N}\rangle_{\mathbb{H}^{3}}=\frac{-\mu(1+\mathcal{O}(1,2 \mu))}{\sqrt{m^{2}+c^{2}(\mu+m)^{2} x^{2 \mu}+\mathcal{O}(1,2 \mu)}} \approx \frac{-\mu}{m} .
$$

Thus, for a $\varphi$ of this type, the length $\langle\varphi, \vec{N}\rangle_{\mathbb{H}^{3}}$ of the normal Jacobi vector field $\varphi^{\perp}$ is bounded and continuous in a neighborhood of the end.

- Suppose $\varphi$ is made by the isometry which is rotation about the $x_{3}$-axis. In this case $\varphi=\left(\overrightarrow{-x_{2}, x_{1}, 0}\right)$ at $\left(x_{1}, x_{2}, x_{3}\right)$. Thus, when $z=x>0$, we have

$$
\langle\varphi, \vec{N}\rangle_{\mathbb{H}^{3}}=\mathcal{O}(1,2 \mu)
$$

and so the length $\langle\varphi, \vec{N}\rangle_{\mathbb{H}^{3}}$ of the normal Jacobi vector field $\varphi^{\perp}$ is bounded and continuous in a neighborhood of the end.

Let $u=\langle\varphi, \vec{N}\rangle_{\mathbb{H}^{3}}$ be the length of the normal variation vector field $\varphi^{\perp}$. In the second and third cases above, $u$ is bounded and continuous at the end asymptotic to the origin in the upper half space model. Hence we can conclude from Harvey 
and Polking's removable singularity theory ([HP], $[\mathrm{P}],[\mathrm{Cho}])$ that $u$ is a weak solution of the Jacobi operator $\Delta u+2 K u=0$ on $\Sigma$, except at the ends where $u$ is not bounded.

So on each visible set counted in $\tilde{v}(M, \varphi), u$ is bounded; and for each visible set counted in $\tilde{v}(M, \varphi)$, the nullity of the visible set with respect to the Dirichlet problem is at least 1 .

The operator $\bar{L}$ on $\Sigma$ has the following properties:

- $\bar{L}$ satisfies the unique continuation property; that is, if two solutions $u$ and $v$ of $\bar{L}=0$ are equal on any open set of $\Sigma$, then they are equal on all of $\Sigma$. This property holds on $\Sigma$ simply because it holds on $\Sigma \backslash\left\{p_{j}\right\}$ (since any weak solution $u$ of $\bar{L} u=0$ on $\Sigma \backslash\left\{p_{j}\right\}$ is also a strong solution on $\Sigma \backslash\left\{p_{j}\right\}$, by elliptic regularity), and because any open set in $\Sigma$ contains an open set of $\Sigma \backslash\left\{p_{j}\right\}$.

- $\bar{L}$ also satisfies a variational characterization of the eigenvalues property. This follows from the standard variational arguments used in the proof of the first three items of Lemma 5.2. The eigenvalues can be characterized as $\lambda_{k}=\min (\mathcal{Q}(u))$, where the functions $u \neq 0$ are any functions that are $L_{d \bar{s}^{2}}^{2}$-perpendicular to the eigenspaces of $\lambda_{1}, \ldots, \lambda_{k-1}$. And those functions $u$ for which $\mathcal{Q}$ attains the minimum $\lambda_{k}$ are precisely the eigenfunctions associated to $\lambda_{k}$.

- Using the two properties above and the variational characterization we used to derive Corollary 5.1, we can conclude that as a domain $\Omega$ increases in size, the eigenvalues (with respect to the Dirichlet problem) must be strictly decreasing.

These properties enable us to conclude that Smale's theorem holds in our setting $[\mathrm{FT}]$.

Smale's result (see [L], Theorem 33). Let $c_{t}$ be a smooth contraction of $\Sigma$ into itself such that

- $c_{0}=$ identity,

- $c_{t}(\Sigma) \subset c_{s}(\Sigma)$ for $t>s$,

- $\lim _{t \rightarrow \infty} \operatorname{Volume}\left(c_{t}\right)=0$.

Then:

$$
\operatorname{Ind}(\Sigma) \geq \sum_{t>0} \operatorname{Nullity}\left(c_{t}\right)
$$

where Nullity $\left(c_{t}\right)$ is the dimension of the space of Jacobi fields on $c_{t}(\Sigma)$ vanishing on the boundary of $c_{t}(\Sigma)$.

Noting that we have shown that $\operatorname{Ind}(\Sigma)=\operatorname{Ind}_{u}(M)$, the proof then follows essentially as in the proofs of Theorem 1 of [Cho]. For the sake of completeness, we include the argument here.

Let $k=v(M, \varphi)$ and $\tilde{k}=\tilde{v}(M, \varphi)$, and let $V_{1}, \ldots, V_{k}$ be the open components of $M \backslash H(M, \varphi)$. Let $\hat{V}_{1}, \ldots, \hat{V}_{k}$ be the open sets of $\Sigma$ corresponding to the 
sets $V_{1}, \ldots, V_{k}$ under the conformal bijection between $\Sigma$ and $M$. After suitably renumbering $\hat{V}_{1}, \ldots, \hat{V}_{k}$, we can exhaust the sets $\hat{V}_{j}$ by a continuous 1-parameter family of shrinking domains $c_{t}(\Sigma), t \in(0, \infty)$ with piecewise smooth boundaries such that $\Sigma \backslash c_{t}(\Sigma) \subset \hat{V}_{1}$ for $t<1$, and $c_{j}(\Sigma)=\hat{V}_{j+1} \cup \cdots \cup \hat{V}_{k}$ for each integer $j=1, \ldots, k-1$, and $c_{t}(\Sigma) \subset \hat{V}_{k}$ for all $t>k-1$. We may assume that $V_{k-\tilde{k}+1}, \ldots, V_{k}$ are the sets that are counted in $\tilde{v}(M, \varphi)$, and that $V_{1}, \ldots, V_{k-\tilde{k}}$ are not counted in $\tilde{v}(M, \varphi)$.

If $\tilde{v}(M, \varphi)<v(M, \varphi)$, it follows that Nullity $\left(c_{t}(\Sigma)\right) \geq 1$ with respect to the Dirichlet problem on $c_{t}(\Sigma)$ when $t=k-\tilde{k}, t=k-\tilde{k}+1, \ldots, t=k-1$. By Smale's Theorem it follows that

$$
\operatorname{Ind}(\Sigma) \geq \tilde{k}
$$

If $\tilde{v}(M, \varphi)=v(M, \varphi)$, it follows that $\operatorname{Nullity}\left(c_{t}(\Sigma)\right) \geq 1$ with respect to the Dirichlet problem on $c_{t}(\Sigma)$ when $t=1, t=2, \ldots, t=k-1$. By Smale's theorem it follows that

$$
\operatorname{Ind}(\Sigma) \geq \tilde{k}-1
$$

Thus, by Lemma 5.3, the theorem is proved.

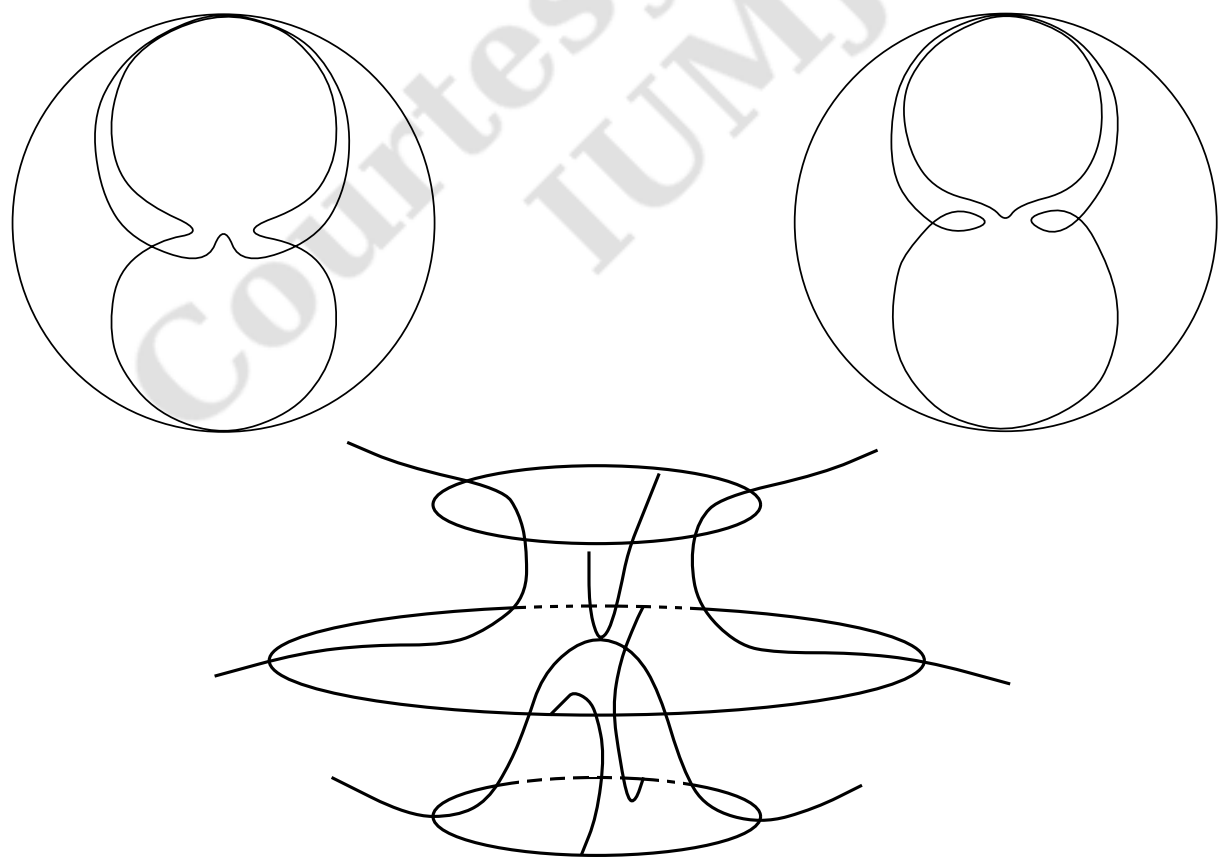

Figure 2: A genus 1 Costa cousin in the Poincaré model: Slices in the $x_{1} x_{3}$-plane and in the $x_{2} x_{3}$-plane. 
We now apply Theorem 7.1 to find lower bounds for the index of several specific examples.

Corollary 7.1 Suppose that $M$ is a constant mean curvature 1 genus $k$ Costa cousin in $\mathbb{H}^{3}$ (as described in [RUY], $M$ is the surface in $\mathbb{H}^{3}$ corresponding to the minimal genus $k$ Costa-Hoffman-Meeks surface). Then $\operatorname{Ind}(M) \geq 2 k$.

Proof. Consider the surface $M$ in the Poincaré model with two ends asymptotic to the point $(0,0,1)$ in the sphere at infinity and one end asymptotic to the point $(0,0,-1)$ in the sphere at infinity (see Figure 2$)$. Let $\varphi$ be the Killing vector field generated by hyperbolic rotation about the $x_{3}$-axis, thus $\varphi$ fixes the two points $(0,0,1)$ and $(0,0,-1)$ in the sphere at infinity. Due to the reflective symmetries of $M$, it is clear that $\tilde{v}(M, \varphi)=v(M, \varphi) \geq 2 k+2$. By Theorem 7.1, the corollary follows.

Using the same $\varphi$ as in the proof above and placing the genus 1 catenoid cousins (as described in $[\mathrm{RS}]$ ) so that their ends are asymptotic to $(0,0,1)$ and $(0,0,-1)$ (see Figure 3$)$, we also have the following corollary.

Figure 3: Half of a genus 1 catenoid cousin (computer graphics by Katsunori Sato of Tokyo Institute of Technology). 


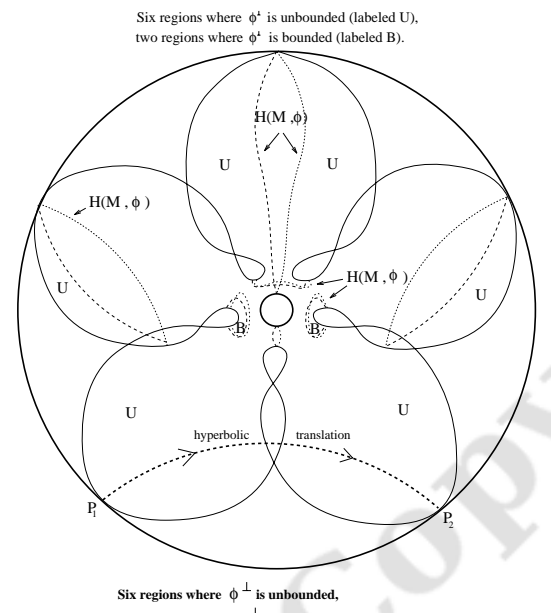

four regions where $\phi^{\perp}$ is bounded.

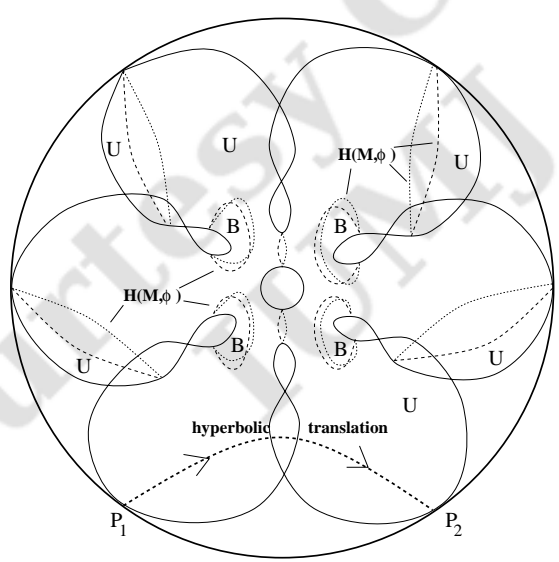

Figure 4: A minimal genus 1 trinoid in $\mathbb{R}^{3}$, and a constant mean curvature 1 genus 1 trinoid cousin in $\mathbb{H}^{3}$.

Corollary 7.2 The genus one catenoid cousins have index at least 2.

The following proposition can be proven by an argument similar to that of Corollary 4 in [Cho]. This result is not stated in [Cho], perhaps only because existence of the minimal genus $1 n$-noid was not known at that time [BR].

Proposition 7.1 The minimal genus 1 n-noid in $\mathbb{R}^{3}$ has index at least $n$ if $n$ is odd, and at least $n-1$ if $n$ is even.

The corollaries above do not require that the surfaces be slight deformations of a corresponding minimal surface, but in the following corollary we will need this assumption. The deformation is described in [RUY]. 
Figure 5: The horizon $H(M, \varphi)$ on genus 1 -noid and 6-noid cousins, as described in the proof of Corollary 7.3.

Corollary 7.3 Let $M$ be a constant mean curvature 1 genus 1 n-noid cousin in $\mathbb{H}^{3}$ that is a slight deformation of a minimal genus 1 n-noid in $\mathbb{R}^{3}$ (see Figure 4). Then $\operatorname{Ind}(M) \geq n-3$ if $n$ is even and $\operatorname{Ind}(M) \geq n-4$ if $n$ is odd.

Proof. Place the surface $M$ in the Poincaré model so that all of its $n$ ends are asymptotic to points in the sphere at infinity where $x_{2}=0$. Let $p_{1}$ and $p_{2}$ be two points in the sphere at infinity such that two adjacent ends of $M$ are asymptotic to $p_{1}$ and $p_{2}$. Let $\varphi$ be a hyperbolic translation fixing $p_{1}$ and $p_{2}$ in the sphere at infinity. When $M$ is a sufficiently small deformation of a minimal genus 1 $n$-noid, we know the behavior of $H(M, \varphi)$ (since we know what the horizon is on the minimal genus $1 n$-noid [Cho]). (See Figure 5.) We can conclude that $n-2=\tilde{v}(M, \varphi)<v(M, \varphi)$ when $n$ is even, and $n-3=\tilde{v}(M, \varphi)<v(M, \varphi)$ when $n$ is odd. Then Theorem 7.1 implies the corollary. 
8. Deformations from minimal surfaces. As stated in Section 3, it was shown in [RUY] that minimal surfaces in $\mathbb{R}^{3}$ can be deformed into corresponding constant mean curvature $c$ surface in $\mathbb{H}^{3}\left(-c^{2}\right)$. And (as described in Section 3) constant mean curvature $c$ surfaces in $\mathbb{H}^{3}\left(-c^{2}\right)$ are equivalent to constant mean curvature 1 surfaces in $\mathbb{H}^{3}$. Most of the known examples of complete constant mean curvature 1 surfaces with finite topology in $\mathbb{H}^{3}$ have been shown to exist only via this deformation. It is for this reason that the following theorem is of interest.

For a minimal surface $M_{0}$, we consider the index $\operatorname{Ind}\left(M_{0}\right)$ to be as defined in [FC]. The index of minimal surfaces is considered without a volume constraint, and this is natural because a volume constraint does not have a physical meaning for minimal surfaces.

Theorem 8.1 If $M_{0}$ is a complete minimal surface with finite total curvature in $\mathbb{R}^{3}$, and $M_{c}$ is a corresponding constant mean curvature $c$ surface in $\mathbb{H}^{3}\left(-c^{2}\right)$, then if $c$ is sufficiently close to zero,

$$
\operatorname{Ind}\left(M_{c}\right) \geq \operatorname{Ind}\left(M_{0}\right)-1 .
$$

It is not known yet if $\operatorname{Ind}\left(M_{0}\right)-1$ is the best possible lower bound for $\operatorname{Ind}\left(M_{c}\right)$ in general, but the embedded catenoid cousins show that the best possible lower bound for $\operatorname{Ind}\left(M_{c}\right)$ cannot be greater than $\operatorname{Ind}\left(M_{0}\right)$ (since the index of a minimal catenoid is 1 and the index of an embedded catenoid cousin is also 1).

Also, it is not possible to have an upper bound for the index of those constant mean curvature $c$ surfaces in $\mathbb{H}^{3}\left(-c^{2}\right)$ which are deformations of minimal surfaces. For example, the deformed Enneper cousin duals have infinite total curvature for all $c \neq 0$, hence also infinite index ([CS]), while the minimal Enneper cousin has index 1 .

Proof. Let $\lambda_{i}(R, c)$ be the $i^{\text {th }}$ eigenvalue of the operator $L$ with respect to the Dirichlet problem on $M_{c} \cap B_{R}(0)$, where

$$
B_{R}(0):=\left\{\left(x_{1}, x_{2}, x_{3}\right) \in \mathbb{R}^{3} \mid x_{1}^{2}+x_{2}^{2}+x_{3}^{2} \leq R^{2}\right\} .
$$

Note that $B_{R}(0)$ is contained in the Poincaré model for $\mathbb{H}^{3}\left(-c^{2}\right)$ if $c$ is sufficiently close to zero, since the Poincaré model has Euclidean radius $1 / c$. Choose a finite $R>0$ large enough that

$$
\operatorname{Ind}\left(M_{0}\right)=\operatorname{Ind}\left(M_{0} \cap B_{R}(0)\right)
$$

with Dirichlet boundary conditions. Thus

$$
\lambda_{1}(R, 0) \leq \ldots \leq \lambda_{k}(R, 0)<0 \leq \lambda_{k+1}(R, 0) .
$$

Let $u_{i}=u_{i}(R, c)$ be the eigenfunction on $M_{c} \cap B_{R}(0)$ corresponding to the eigenvalue $\lambda_{i}(R, c)$.

Note that for any finite value of $R, \lambda_{i}(R, c)$ is continuous in $c$. We can see this from the variational characterization of the eigenvalues ([Be], p. 60): 


$$
\lambda_{i}(R, c)=\inf \left\{\frac{\int_{M_{c} \cap B_{R}(0)} u L u d A}{\int_{M_{c} \cap B_{R}(0)} u^{2} d A} \mid u \in C_{0}^{\infty}\left(M_{c} \cap B_{R}(0)\right), u \neq 0,\right.
$$

Since $M_{c} \cap B_{R}(0)$ is compact and $L$ is uniformly continuous on a compact region, we know that this infimum will vary continuously in $c$. Therefore, for $c$ sufficiently close to 0 , we still have

$$
\lambda_{1}(R, c) \leq \ldots \leq \lambda_{k}(R, c)<0
$$

Thus we have $\operatorname{Ind}_{u}\left(M_{c}\right) \geq \operatorname{Ind}_{u}\left(M_{c} \cap B_{R}(0)\right) \geq \operatorname{Ind}\left(M_{0} \cap B_{R}(0)\right)=\operatorname{Ind}\left(M_{0}\right)$, and by Lemma 5.3 we have $\operatorname{Ind}\left(M_{c}\right) \geq \operatorname{Ind}\left(M_{0}\right)-1$.

Corollary 8.1 For sufficiently small (constant mean curvature c) deformations $M$ in $\mathbb{H}^{3}\left(-c^{2}\right)$ from minimal surfaces in $\mathbb{R}^{3}$, we have the following lower bounds for index:

- If $M$ is a genus 0 -noid cousin, then $\operatorname{Ind}(M) \geq 2 n-4$.

- If $M$ is a genus $1 n$-noid cousin, then $\operatorname{Ind}(M) \geq n-2$.

- If $M$ is a genus $k$ Costa cousin with $k \leq 37$, then $\operatorname{Ind}(M) \geq 2 k+2$.

At first, the second part of this lemma may seem like a stronger result than Corollary 7.3, but in Corollaries 8.1 and 7.3, we do not know how large a deformation is possible. It is possible that Corollary 7.3 will allow larger deformations than Corollary 8.1 will allow. Hence we cannot say that Corollary 8.1 is a stronger result than Corollary 7.3.

Proof. Nayatani [N1] showed that the minimal genus $0 n$-noid in $\mathbb{R}^{3}$ has index $2 n-3$. By Proposition 7.1, the minimal genus $1 n$-noid in $\mathbb{R}^{3}$ has index at least $n-1$. Nayatani [N2] also showed that the minimal genus $k$ Costa surface in $\mathbb{R}^{3}$ has index $2 k+3$ for all $k \leq 37$. By Theorem 8.1 , the corollary follows.

9. Appendix: Asymptotic behavior of ends. Let $D \backslash\{0\}$ be the unit disk in the plane with the origin removed. Let $\Phi: D \backslash\{0\} \rightarrow \mathbb{H}^{3}$ be a constant mean curvature 1 , finite total curvature surface with a complete regular end at 0 . We can take the secondary Gauss map to be $G=z^{\mu} \hat{G}$, and we can take the Hopf differential $Q$ so that $Q /(d G)=\omega d z=z^{\nu} \hat{\omega} d z$ where $\mu, \nu \in \mathbb{R}$ and $\hat{G}, \hat{\omega}$ are holomorphic and $\hat{G}(0) \neq 0, \hat{\omega}(0) \neq 0$ [UY1]. As in the proof of Lemma 4.2, we 
may assume $\mu>0$. The fact that the end is regular implies that $\operatorname{ord}_{0}(Q) \geq-2$ $[\mathrm{By}]$, hence

$$
Q=\left(\frac{q_{-2}}{z^{2}}+\frac{q_{-1}}{z}+\cdots\right)(d z)^{2} .
$$

The leading coefficient $q_{-2}$ may or may not be zero. Completeness implies that $\nu \leq-1$ (this follows just by considering $d s^{2}$ ), and the fact that $Q$ is meromorphic implies that $\mu+\nu \in \mathbb{Z}$. Finding a solution

$$
F^{-1}=\left(\begin{array}{cc}
A & B \\
C & D
\end{array}\right)
$$

in the Weierstrass representation (Lemma 3.2) for this surface, we have that $A$ and $C$ satifsy (see [UY1])

$$
X^{\prime \prime}-\frac{\omega^{\prime}}{\omega} X^{\prime}-\omega G^{\prime} X=0,
$$

(where $/$ denotes $d / d z$ ) and $B$ and $D$ satifsy

$$
Y^{\prime \prime}-\frac{\left(G^{2} \omega\right)^{\prime}}{G^{2} \omega} Y^{\prime}-\omega G^{\prime} Y=0 .
$$

The indicial equations of the second order equations above are

$$
t^{2}-(\nu+1) t-q_{-2}=0 \quad \text { and } \quad t^{2}-(2 \mu+\nu+1) t-q_{-2}=0 .
$$

The differences of solutions for the indicial equations are $m_{1}=\sqrt{(\nu+1)^{2}+4 q_{-2}}$ and $m_{2}=\sqrt{(2 \mu+\nu+1)^{2}+4 q_{-2}}$. By results in [UY1], if the end is well defined, then $m_{1}, m_{2} \in \mathbb{Z}^{+}$, and the end is embedded if and only if $m:=\min \left(m_{1}, m_{2}\right)=$ 1 .

On page 626 of [UY1] the end $\Phi$ is classified into three possible cases.

- $\mu=0, m_{1}=m_{2}=m$.

- $\mu \neq 0, \operatorname{ord}_{0}(Q)=-2, m_{1}=m_{2}=m$.

- $\mu \neq 0, \operatorname{ord}_{0}(Q) \geq-1, m_{1}=-(\nu+1), m_{2}=2 \mu+\nu+1, m_{2}-m_{1}=$ $2\left(\operatorname{ord}_{0}(Q)+2\right)>0$.

In all three cases $m=m_{1}$.

As we saw in the proof of Lemma 4.2, we may replace $\mathrm{F}$ by $B F$ for some $B \in$ $\mathrm{SU}(2)$ so that $\mu>0$. Thus the first case above always reduces to the second case (if $\operatorname{ord}_{0}(Q)=-2$ ) or the third case (if $\left.\operatorname{ord}_{0}(Q)>-2\right)$. Hence we only need to consider Cases 2 and 3 .

In Case 2, we say that we have a catenoid cousin type end. In Case 3 we say that we have a horosphere type end. Let $\mathcal{O}\left(z^{\alpha}\right)$ denote any complex valued function $f$ such that $\lim \sup _{z \rightarrow 0}|f| / z^{\alpha}$ is bounded. 
Lemma 9.1 At a catenoid cousin type end, $\mu \neq m$ and $F^{-1}$ can be locally represented as

$$
F^{-1}=\frac{1}{\sqrt{\mu m}}\left(\begin{array}{ll}
\frac{\mu+m}{2} z^{(-\mu+m) / 2}(1+\mathcal{O}(z)) & \frac{\mu-m}{2} z^{(\mu+m) / 2}(1+\mathcal{O}(z)) \\
\frac{\mu-m}{2} z^{(-\mu-m) / 2}(1+\mathcal{O}(z)) & \frac{\mu+m}{2} z^{(\mu-m) / 2}(1+\mathcal{O}(z))
\end{array}\right)
$$

Proof. In Case 2, $q_{-2} \neq 0$ and $\mu+\nu=-1$ and $m^{2}=m_{1}^{2}=(\nu+1)^{2}+4 q_{-2}$. Hence $(-\mu)^{2}=(\nu+1)^{2} \neq m^{2}$, so $\mu \neq m$.

By Lemma 5.3 of [UY1],

$$
\Delta \cdot F^{-1}=\frac{1}{\sqrt{\mu m}}\left(\begin{array}{cc}
\frac{\mu+m}{2} z^{(-\mu+m) / 2} a(z) & \frac{\mu-m}{2} z^{(\mu+m) / 2} b(z) \\
\frac{\mu-m}{2} z^{(-\mu-m) / 2} c(z) & \frac{\mu+m}{2} z^{(\mu-m) / 2} d(z)
\end{array}\right),
$$

where $a(z), b(z), c(z), d(z)$ are holomorphic and nonzero at $z=0$. Since $\Delta \in$ $\mathrm{SL}(2, \mathbb{C})$ only represents an isometry of $\mathbb{H}^{3}$ [UY1], we can assume $\Delta$ is the identity matrix. By doing the transformation $z \rightarrow(G(0))^{-1 / \mu} z$, we have $G=z^{\mu}\left(1+g_{1} z+\cdots\right)$. By equation (3.1) we know $g=d A /(d C)=d B /(d D)$, and computing $g$ we find that $g \approx z^{m}$ and $a(0) d(0)=b(0) c(0)$. Since $\operatorname{det}\left(F^{-1}\right)$ $=1$, we have $a(0) d(0)=b(0) c(0)=1$. Since $G=-d B / d A=-d D / d C$, we have that $a(0)=b(0)$ and $c(0)=d(0)$. Doing an isometry of $\mathbb{H}^{3}$ so that

$$
F^{-1} \rightarrow\left(\begin{array}{cc}
\frac{1}{a(0)} & 0 \\
0 & a(0)
\end{array}\right) F^{-1}
$$

we have proved the lemma.

Lemma 9.2 At a horosphere type end, $F^{-1}$ can be locally represented as

$$
F^{-1}=\left(\begin{array}{cc}
1+\mathcal{O}(z) & \mathcal{O}\left(z^{2 \mu+\nu+1}\right) \\
z^{\nu+1}(1+\mathcal{O}(z)) & 1+\mathcal{O}(z)
\end{array}\right)
$$

Proof. Note that for Case 3, we have $\mu \geq-\nu \geq 2, \mu, \nu \in \mathbb{Z}$. By Lemma 5.3 of [UY1],

$$
\Delta \cdot F^{-1}=\left(\begin{array}{cc}
a(z) & z^{2 \mu+\nu+1} b(z) \\
z^{\nu+1} c(z) & d(z)
\end{array}\right),
$$

where $a(z), b(z), c(z), d(z)$ are holomorphic and nonzero at $z=0$. Again, $\Delta$ only represents an isometry of $\mathbb{H}^{3}$. We may assume $\Delta_{11}=\zeta \in \mathbb{C} \backslash\{0\}, \Delta_{22}=\zeta^{-1}$, $\Delta_{12}=\Delta_{21}=0$, and then for any value of $\zeta$ the end is still asymptotic to the 
origin in the upper half space model. With an appropriate choice of $\zeta$, we can conclude that $a(0)=d(0)$. We can then rewrite $\Delta \cdot F^{-1}$ simply as $F^{-1}$. Since $\operatorname{det}\left(F^{-1}\right)=1$, we have $a(0)=d(0)=1$. Transforming $z \rightarrow(c(0))^{-1 /(\nu+1)} z$, we have the lemma.

The point

$$
F^{-1} \overline{F-1}^{t}=\left(\begin{array}{cc}
A & B \\
C & D
\end{array}\right)\left(\begin{array}{cc}
\bar{A} & \bar{C} \\
\bar{B} & \bar{D}
\end{array}\right)
$$

in the Hermitian model corresponds to the point

$$
\frac{(\operatorname{Re}(A \bar{C}+B \bar{D}), \operatorname{Im}(A \bar{C}+B \bar{D}), 1)}{C \bar{C}+D \bar{D}}
$$

in the upper half space model. So the catenoid cousin type end is

$$
\begin{aligned}
& \frac{\mu+m}{\mu-m}\left(\operatorname{Re}\left(z^{m}\right)\left(1+\mathcal{O}\left(|z|^{\min (1,2 \mu)}\right)\right), \operatorname{Im}\left(z^{m}\right)\left(1+\mathcal{O}\left(|z|^{\min (1,2 \mu)}\right)\right)\right. \\
& \left.\frac{4 \mu m}{\mu^{2}-m^{2}}|z|^{\mu+m}\left(1+\mathcal{O}\left(|z|^{\min (1,2 \mu)}\right)\right)\right)
\end{aligned}
$$

in the upper half space model. $\mathcal{O}\left(|z|^{\alpha}\right)$ denotes any real valued function such that $\limsup _{z \rightarrow 0}|f| /|z|^{\alpha}$ is finite. The horosphere end is

$$
\left(\operatorname{Re}\left(z^{m}\right)(1+\mathcal{O}(|z|)), \operatorname{Im}\left(z^{m}\right)(1+\mathcal{O}(|z|)),|z|^{2 m}(1+\mathcal{O}(|z|))\right)
$$

in the upper half space model, where $m=-\nu-1$.

Lemma 9.3 An end of the form

$$
\left(c_{1} \operatorname{Re}\left(z^{m}\right)\left(1+\mathcal{O}\left(|z|^{\alpha}\right)\right), c_{1} \operatorname{Im}\left(z^{m}\right)\left(1+\mathcal{O}\left(|z|^{\alpha}\right)\right), c_{2}|z|^{\mu+m}\left(1+\mathcal{O}\left(|z|^{\alpha}\right)\right)\right)
$$

can be written in the form

$$
\left(\operatorname{Re}\left(z^{m}\right), \operatorname{Im}\left(z^{m}\right), c_{3}|z|^{\mu+m}\left(1+\mathcal{O}\left(|z|^{\alpha}\right)\right)\right) .
$$

Proof. There exists $\tilde{z} \approx z$ so that $z^{m}\left(1+\mathcal{O}\left(|z|^{\alpha}\right)\right)=\tilde{z}^{m}$, by the Weierstrass preparation theorem. If follows that $(z / \tilde{z})^{m}-1 \in \mathcal{O}\left(|z|^{\alpha}\right)$, and therefore $\lim _{z \rightarrow 0} z / \tilde{z}=\lim _{z \rightarrow 0}(z / \tilde{z})^{\alpha}=1$. Also, we have $z^{m}-\tilde{z}^{m} \in \mathcal{O}\left(|z|^{\alpha}\right) z^{m}$, so $m z^{m-1}(z-\tilde{z}) \approx z^{m}-\tilde{z}^{m} \in \mathcal{O}\left(|z|^{\alpha+m}\right)$ by the mean value theorem, so $z-\tilde{z} \in \mathcal{O}\left(|z|^{\alpha+1}\right)$. Now we have

$$
\begin{aligned}
\left.\left|c_{2}\right| z\right|^{\mu+m}\left(1+\mathcal{O}\left(|z|^{\alpha}\right)\right)-c_{2}|\tilde{z}|^{\mu+m} \mid & \leq\left. c_{2}|| z\right|^{\mu+m}-|\tilde{z}|^{\mu+m}+|z|^{\mu+m} \mathcal{O}\left(|z|^{\alpha}\right) \mid \\
& \leq\left. c_{2}|| z\right|^{\mu+m}-|\tilde{z}|^{\mu+m} \mid+\mathcal{O}\left(|z|^{\alpha+\mu+m}\right) \\
& \leq c_{2}\left|z^{\mu+m}-\tilde{z}^{\mu+m}\right|+\mathcal{O}\left(|z|^{\alpha+\mu+m}\right)
\end{aligned}
$$

(triangle inequality) 


$$
\begin{aligned}
& \approx c_{2}(\mu+m)|z|^{\mu+m-1}|z-\tilde{z}|+\mathcal{O}\left(|z|^{\alpha+\mu+m}\right) \\
& =|z|^{\mu+m-1} \mathcal{O}\left(|z|^{\alpha+1}\right)+\mathcal{O}\left(|z|^{\alpha+\mu+m}\right) \\
& =\mathcal{O}\left(|z|^{\alpha+\mu+m}\right) .
\end{aligned}
$$

So we have that $c_{2}|z|^{\mu+m}\left(1+\mathcal{O}\left(|z|^{\alpha}\right)\right)=c_{2}|\tilde{z}|^{\mu+m}+\mathcal{O}\left(|z|^{\alpha+\mu+m}\right)$ and therefore $c_{2}|z|^{\mu+m}\left(1+\mathcal{O}\left(|z|^{\alpha}\right)\right)=c_{2}|\tilde{z}|^{\mu+m}\left(1+\mathcal{O}\left(|\tilde{z}|^{\alpha}\right)\right)$. So we can rewrite our parametrization as

$$
\left(c_{1} \operatorname{Re}\left(\tilde{z}^{m}\right), c_{1} \operatorname{Im}\left(\tilde{z}^{m}\right), c_{2}|\tilde{z}|^{\mu+m}\left(1+\mathcal{O}\left(|\tilde{z}|^{\alpha}\right)\right)\right) .
$$

Then making the transformation $\tilde{z}=c_{1}^{-1 / m} z$, we have finished the proof.

So a regular end of finite total curvature is of the form

$$
\left(\operatorname{Re}\left(z^{m}\right), \operatorname{Im}\left(z^{m}\right), c|z|^{\mu+m}\left(1+\mathcal{O}\left(|z|^{\min (1,2 \mu)}\right)\right)\right)
$$

Note that when we describe the end as a graph like this, the immersion is no longer conformal. The end is embedded if and only if $m=1$ [UY1].

Addendum. Lucas Barbosa and Pierre Berard have recently announced a result that would imply $\operatorname{Ind}(M)=\operatorname{Ind}_{u}(M)$ for every case in this paper. Using their result, one could strengthen Lemma 5.3 by excluding the $\operatorname{Ind}(M)=$ $\operatorname{Ind}(\Sigma)-1$ case. This would result in corresponding strengthenings of Theorem 6.1 (the $2[\mu]$ case could be excluded), Theorem $7.1(\tilde{v}(M, \varphi)-1$ and $\tilde{v}(M, \varphi)-2$ could be replaced with $\tilde{v}(M, \varphi)$ and $\tilde{v}(M, \varphi)-1$, respectively), Corollary 7.1 ( $2 k$ could be replaced with $2 k+1)$, Corollary 7.2 (2 could be replaced with 3$)$, Corollary 7.3 ( $n-3$ and $n-4$ could be replaced with $n-2$ and $n-3$, respectively), Theorem $8.1\left(\operatorname{Ind}\left(M_{0}\right)-1\right.$ could be replaced with $\left.\operatorname{Ind}\left(M_{0}\right)\right)$, and Corollary 8.1 $(2 n-4, n-2$, and $2 k+2$ could be replaced with $2 n-3, n-1$, and $2 k+3$, respectively).

\section{REFERENCES}

[Ad] R. Adams, Sobolev Spaces, Academic Press (1975).

[BCE] J. L. Barbosa, M. Do CARmo, Eschenburg. Stability of Hypersurfaces of Constant Mean Curvature in Riemannian Manifolds. Math. Z. 197 (1988), 123-138.

[Be] P. Berard. Spectral Geometry: Direct and Inverse Problems. Lecture Notes in Math. 1207, Springer-Verlag (1986).

[BCS] P. Berard, M. P. Do Carmo, W. Santos. The Index of Constant Mean Curvature Surfaces in Hyperbolic 3-Space. Math. Z. 224 (1997), 313-326.

[BR] J. Berglund, W. Rossman. Minimal Surfaces with Catenoid Ends. Pacific Journ. Math. 171(2) (1995), 353-371. 
[By] R. BRyAnt. Surfaces of Mean Curvature One in Hyperbolic Space. Astérisque, 154-155 (1987), 321-347.

[CP] M. P. do CARMo, C. K. Peng. Stable minimal surfaces in $\mathbb{R}^{3}$ are planes, Bull. of the Amer. Math. Soc. 1 (1979), 903-906.

[CS] M. P. Do CARmo, A. M. Silveira. Index and total curvature of surfaces with constant mean curvature, Proc. Amer. Math. Soc. 110 (1990), 1009-1015.

[Che] S. S. Chern. Minimal submanifolds in a Riemannian manifold, Dept. of Math. Technical Report 19, Lawrence, Kansas: Univ. of Kansas (1968).

[Cho] J. ChoE. Index, vision number, and stability of complete minimal surfaces, Arch. Rat. Mech. Anal. 109 (1990), 195-212.

[EK] N. EJIRI, M. Kotani. Index and flat ends of minimal surfaces, Tokyo J. of Math. 16 (1993), 37-48.

[FC] D. FischeR-COLBRIE. On complete minimal surfaces with finite Morse index in three manifolds, Invent. Math. 82 (1985), 121-132.

[FT] H. FrID, F. J. ThAYER. An absolute version of the Morse index theorem and its applications to hypersurfaces of constant mean curvature, Bol. Soc. Bras. Mat. 20 (1990), 59-68.

[G] R. Gulliver. Index and total curvature of complete minimal surfaces, Proc. Symp. Pure Math. 44 (1986), 207-211.

[GT] D. Gilbarg, N. S. Trudinger. Elliptic Partial Differential Equations of Second Order, Second Edition. Grundlehren der Mathematischen Wissenschaften 224, A Series of Comprehensive Studies in Mathematics, Springer-Verlag (1983).

[H] Hormander. The Analysis of Linear Partial Differential Operators III. Grundlehren der Mathematischen Wissenschaften 274, A Series of Comprehensive Studies in Mathematics, Springer-Verlag (1980).

[HP] R. HARvey, J. Polking. Removable singularities of solutions of linear partial differential equations. Acta Math. 125 (1970), 39-56.

[HT] D. Hulin, M. Troyanov. Prescribing Curvature on Open Surfaces. Math. Ann. 293 (1992), 277-315.

[KKMS] N. KorevaAr, R. Kusner, W. Meeks, B. Solomon. Constant Mean Curvature Surfaces in Hyperbolic Space. American J. Math. 114 (1992), 1-43.

[L] H. B. Lawson. Lectures on Minimal Surfaces, Publish or Perish Press, Berkeley, CA (1980).

[MR] S. Montiel, A. Ros. Schrödinger operators associated to a holomorphic map, in: 'Global Differential Geometry and Global Analysis,' Lect. Notes in Math. \# 1481, Berlin (1991), 147-174.

[N1] S. NAYATANI. Lower bounds for the morse index of complete minimal surfaces in Euclidean 3-space, Osaka J. Math. 27 (1990), 453-464.

[N2] S. Nayatani. Morse index of complete minimal surfaces: The Problem of Plateau, ed. by Th. M. Rassias, World Scientific Publishing, Singapore (1992), 181-189.

[O] R. Osserman. A Survey of Minimal Surfaces, Dover Publications, New York (1969).

[P] J. C. Polking. A survey of removable singularities, Math. Sci. Rex. Inst. Publications 2 (1983), 261-292.

[RS] W. Rossman, K. Sato. Constant mean curvature surfaces with two ends in hyperbolic space. J. Exp. Math. 7(1) (1998). 
[RUY] W. Rossman, M. Umehara, K. YAmada. Irreducible constant mean curvature 1 surfaces in hyperbolic space with positive genus. Tohoku Math. J. 49 (1997), 449-484.

[Si] A. M. SilveIRA. Stability of complete noncompact surfaces with constant mean curvature, Math. Ann. 277 (1987), 629-638.

[T] M. Troyanov. Prescribing Curvature on Compact Surfaces with Conical Singularities. Trans. Amer. Math. Soc. 324(2) (1991), 793-821.

[UY1] M. Umehara, K. Yamada. Complete Surfaces of Constant Mean Curvature One in the Hyperbolic 3-Space. Ann. of Math. 137 (1993), 611-638.

[UY2] M. Umehara, K. Yamada. A Parametrization of the Weierstrass Formulae and Perturbation of Some Complete Minimal Surfaces of $\mathbb{R}^{3}$ into the Hyperbolic 3-Space. J. Reine Angew, Math. 432 (1992), 93-116.

[UY3] M. Umehara, K. Yamada. Surfaces of Constant Mean Curvature $c$ in $\mathbb{H}^{3}\left(-c^{2}\right)$ with Prescribed Hyperbolic Gauss Map. Math. Ann. 304 (1996), 203-224.

[UY4] M. Umehara, K. Yamada. A Duality on Constant Mean Curvature Surfaces in Hyperbolic Space, and a Hyperbolic Analogue of the Osserman Inequality. Tsukuba J. Math. 21(1) (1997), 229-237.

LEVI LOPES DE LiMA

Department of Mathematics

Universidade Federal do Ceará

Fortaleza, Brasil

E-MAIL: levi@mat.ufc.br

WAYNE Rossman

Graduate School of Mathematics

School of Engineering

Kyushu University

Fukuoka 812-81, JAPAN

E-MAIL: wayne@math.kyushu-u.ac.jp

Received: May 21st, 1997; revised: February 2nd, 1998. 